\title{
Bi-objective Optimal Design of a Damage-Tolerant Multifunctional Battery System
}

\author{
Trung N. Nguyen ${ }^{1}$, Thomas Siegmund ${ }^{1}$, Waterloo Tsutsui ${ }^{2}$, Hangjie Liao ${ }^{2}$, Wayne Chen $^{2}$ \\ ${ }^{1}$ School of Mechanical Engineering, Purdue University, West Lafayette, IN 47907, USA \\ ${ }^{2}$ School of Aerospace and Aeronautical Engineering, Purdue University, West Lafayette, IN 47907, \\ USA
}

\begin{abstract}
In current electric vehicles (EVs), battery systems only serve the role of electrical energy storage. Heavy protecting structures surround the batteries. To enhance the overall performance of EVs one desires multifunctional battery systems with structural and energy storage functionalities. Our research centers on the design methodology to enable such multifunctional material systems. An architectured multifunctional battery-structure material system, namely the Cellular Battery Assembly (CBA), is introduced. The CBA is composed of cylindrical battery cells surrounded by hollow tubes. The CBA inherits features of the energy absorbing characteristics of periodic cellular materials while maintaining an electrical energy storage. The energy absorbing mechanism is activated when intentional collapse of hollow tubes dissipates energy and protects battery units from high compressive stress. Depending on loading conditions to be applied, the multi-functionalities of CBA can be optimized by morphological or dimensional control of the building blocks through a multi-objective optimization process. The present study focuses on a multi-objective optimization design process to achieve optimal configurations in specific applications where energy storage and energy absorption are objective functions to be maximized simultaneously. Results show that CBA can possess energy absorption performance that compares well to other energy absorbing materials while a desirable energy storage capability is maintained.
\end{abstract}

Keywords: architectured material system, multifunctionality, energy storage, energy absorption, Pareto-optimality 


\section{INTRODUCTION}

Electrification of transportation systems is an emerging global trend [1, 2]. Battery systems play a key role in related technical advances to engineering efficient and safe vehicle solutions. One aspect of battery systems in transportation applications is safety, and design against thermal runaway caused by mechanical abuse such as a vehicle crash has been identified as a key engineering aspect. Only if battery safety can adequately be addressed, will design engineers be able to create attractive and efficient electric vehicles. Thereby, the concern is that the failure of one or multiple battery cells results in an uncontrollable spread of failure throughout the entire battery system [3]. Consequently, several research groups have been investigating approaches to damage tolerant batteries, see e.g. [4, 5]. The present study focusses on damage tolerant battery systems [6], whereby damage tolerance is imparted by (micro)-structural features of the battery pack rather than by alternations to the battery composition. Such a damage tolerant battery system is inherently multifunctional, as both energy storage and damage tolerance criteria have to be addressed simultaneously. The battery system of interest here can be considered as a hybrid cellular material where one group of cells is filled with batteries.

There has been significant recent interest in approaches considering the integration of mechanical structural features jointly with electrical energy capabilities to create multifunctional batterystructure material systems [2, 3]. Such multifunctional battery systems [7, 8] whose overall performance can be improved through weight and/or volume savings [9]. A multifunctional approach [3] has been applied in the engineering of battery units for ground [10], air and space vehicles [11]. Multifunctional power structures that merge the load carrying capabilities of traditional structural components with other stand-alone functions leading to a significant mass reduction have been proposed in spacecraft engineering and satellite design $[11,12]$. Flight endurance time of unmanned air-vehicle can be increased by integrating Li-ion cells into wing structural skin which results in a multifunctional wings of reduced weight [8]. Structural battery systems have been investigated to introduce benefits of dual functionality when using a structural power source on EVs [3] or prismatic cellular materials as load-bearing heat exchangers [9]. Proper design approaches for multifunctional battery-structure system are still being sought.

In this study we address the questions of how to effectively integrate functionalities of the two components, battery and energy dissipating structure, and how to achieve optimal systems configurations. The general multi-material design procedure [13] is adopted and further expanded in detailed steps. Being a design process, the procedure and method involve a series of decision making steps. Therefore, a set of performance metrics is required to set criteria based on which selections and decisions can be made. A multi-objective optimization design tool is introduced to enable a thorough exploration not only in the design space but also in the functional space. Following steps in the suggested design procedure, a multifunctional battery-structure material system, namely Cellular Battery Assembly (CBA), is proposed and optimized to achieve high energy storage and energy absorption simultaneously.

The concept of multi-material design is applied to combine two constituents: an energy storage and an energy absorbing material. Particularly, CBA is constructed by assembling Li-ion battery cells and sacrificial hollow tubes following an architectured pattern. CBA inherits features of the energy absorbing characteristics of cellular solids [14] while providing energy storage in battery units. The resulting characteristics of CBA cannot be achieved by either battery cells or hollow tubes alone, and the combination of the two aspects defines a multifunctional structure-battery material system. Its properties lie in the unreachable area for any single constituent component, Figure 1. The integrated self-protection feature of CBA is proposed to enable the installation of energy storage in crumple zones of EVs. This provides novel options for energy storage in EVs. CBA can be considered as an architectured periodic cellular material system. Optimal configurations of CBA can be obtained via mesostructured arrangement and selection of volume fraction of each components by dimensional adjustments. In doing so, the specific energy storage (SES), the stored energy-to-mass ratio and the specific energy absorption (SEA), the absorbed energy-to-mass ratio, are selected to be objective functions to be maximized. Design constraints emerge from the requirement of battery safety. 
Consequently, the design procedure involves solving a multi-objective optimization problem of the two conflicting objective functions: maximize SES and SEA simultaneously. Conditions of battery safety obtained from failure tests of single battery cells motivate the design constraint. An evolutionary approach, the predator-prey genetic algorithm (PPGA), is applied to find Pareto optimal solutions of the multi-objective optimization problem. Elaborate mathematical details of Paretooptimality are available in [15-17]. Further relevant information about the metaheuristic optimization technique can be found elsewhere [18, 19]. Finally, a best final configuration is selected using a selection criterion which suggests a preferred configuration among solutions in the Pareto optimal set. Such selection criterion should be provided to avoid biasing a designer from his or her subjectivity. A method using predefined exchange constants to relate objectives to a value function which has the units of currency can be applied [20]. The selection criterion used in this study, however, is related to mechanics of the system rather than using a value function.

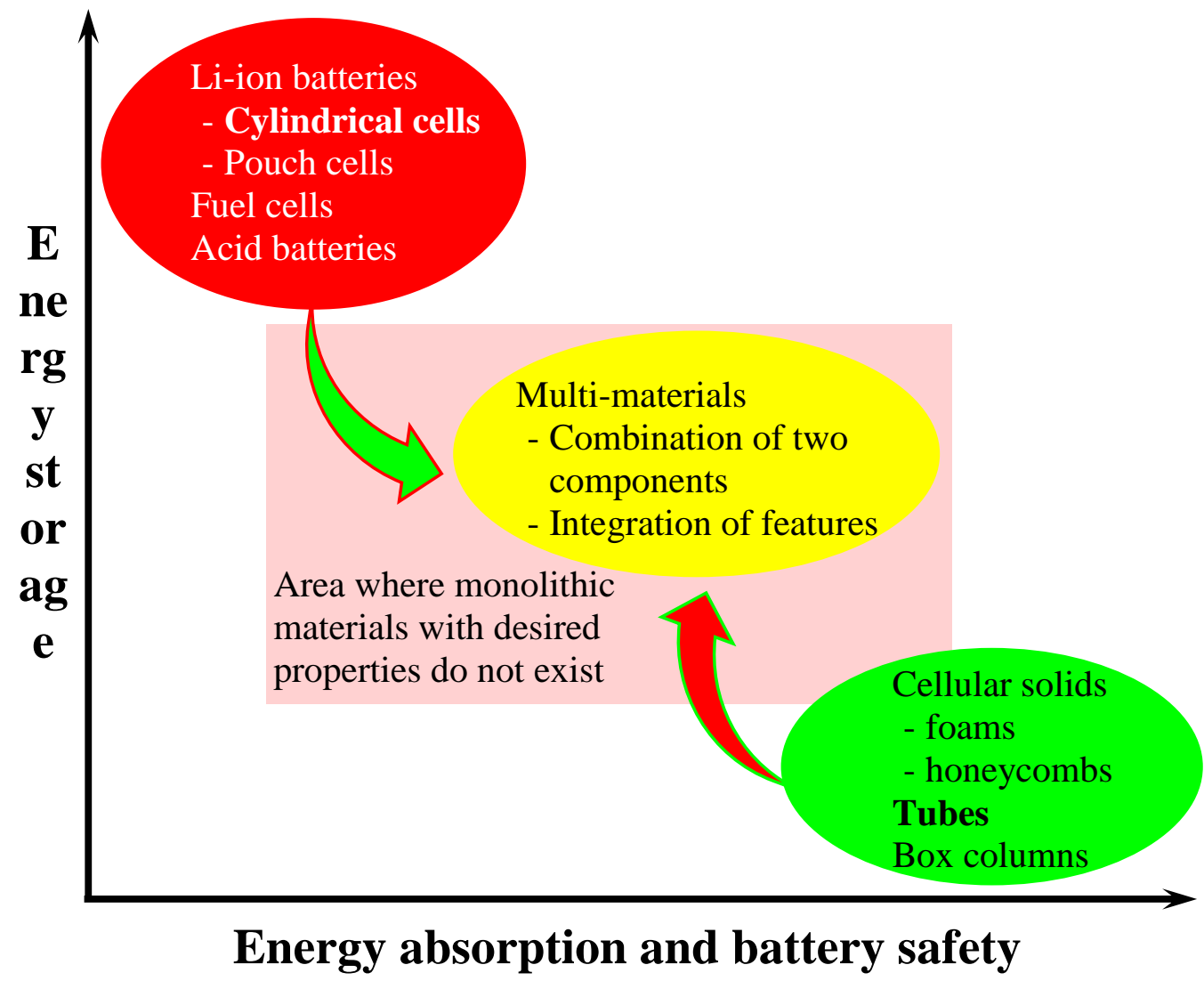

Figure 1. The concept of design for multifunctional material systems.

\section{DESIGN PROCEDURE AND METHOD}

\subsection{Design procedure}

A multifunctional material system design is an outcome of decision making steps including the selection of constituents, architecture, and the interface between constituents. Discussions of the selections can be found in the literature [13, 20-22]. Provided that the design requirements and constraints are well defined, the steps of the design procedure are shown in Figure 2. The concept of a multifunctional battery material system is introduced to meet the design requirements, i.e. achieving high energy storage capacity, effective protection and being lightweight, and constraints, i.e. keeping battery safe. Figure 3 depicts the multifunctionality of CBA in the performance space of energy absorption and energy storage. Then, the design is refined using a series of decision making steps, from the selection of constituents to the architecture and their integration. The selections of metrics for the performance evaluation, and the design tools for further refinement of the design are problem dependent and thus require critical consideration. The design process involves a multi- 
objective optimization step to find optimal volume fraction of the two components to maximize the functionalities simultaneously. Finally, additional information related to a preferred working condition and/or reduced cost can be considered to decide a final configuration.

\subsection{Optimization method}

\section{The Predator-Prey Genetic Algorithm (PPGA)}

The design of multifunctional material systems involves contradicting objectives. An improvement in one objective often leads to a deterioration in others and vice versa. This leads to a multi-objective Pareto-optimal problem. Therefore, a multi-objective optimization tool is required to find optimal configurations of CBA by solving the Pareto-optimal problem.

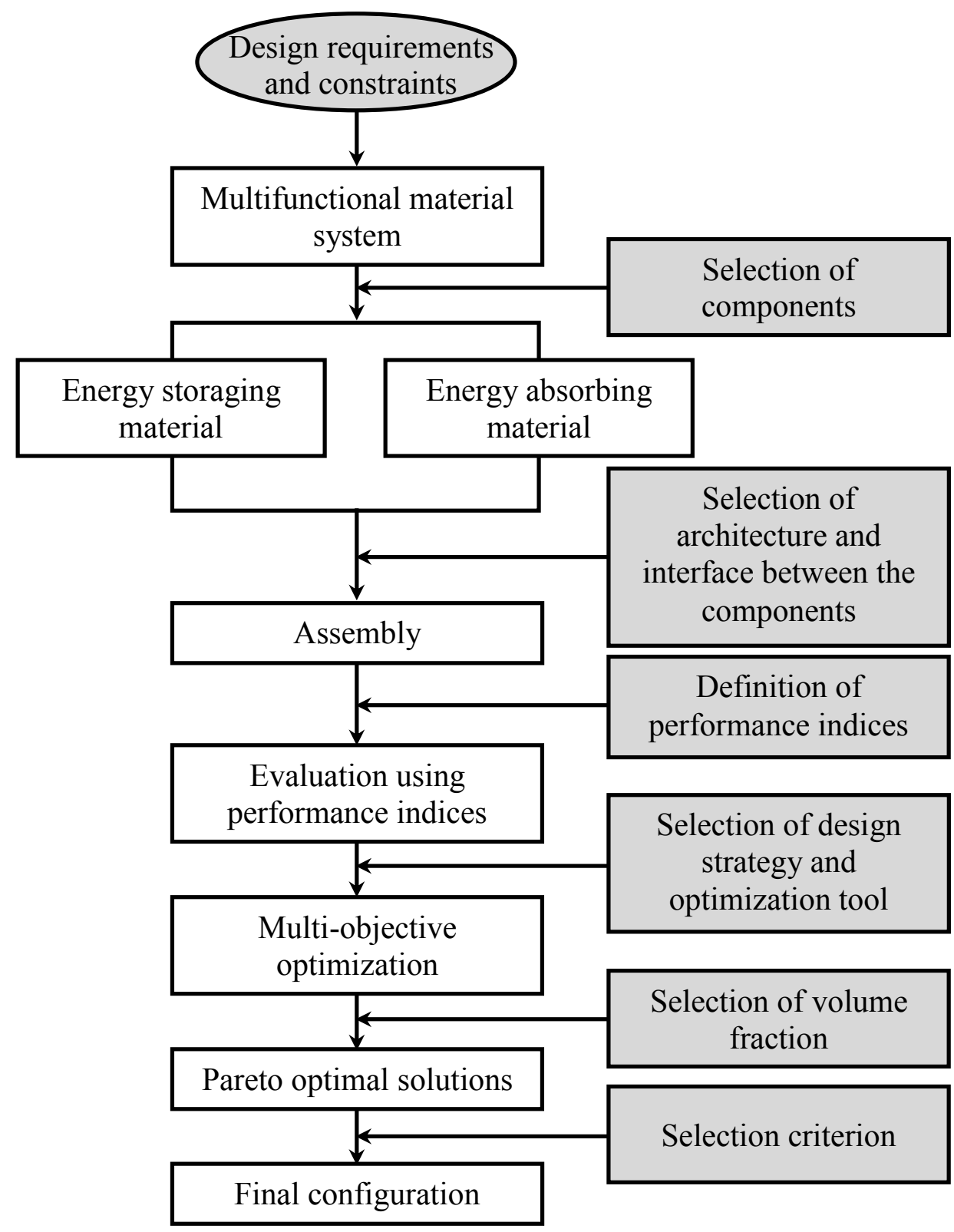

Figure 2. Design procedure for an optimal multifunctional battery-structure material system.

Optimal configurations of CBA are targeted to maximize the dual-functionalities, energy storage and energy dissipation capabilities, while satisfying the associated constraints. This leads to a biobjective optimization problem. As will be seen later, one of the objectives, the energy dissipation, cannot be expressed in an explicit mathematical expression. The absence of an explicit expression limits the use of a gradient-based approach, which always requires proper computations of the 
gradient of the objective functions. Therefore, a gradient-free algorithm is an appropriate choice for such problem.

A PPGA is applied to find optimal configurations that achieve both objectives simultaneously. Being a stochastic global search method, the PPGA loosely mimics the evolutionary process in nature following Darwin's principle of survival of the fittest. The solution strategy involves two distinct populations, prey and predators. The prey represent a set of possible solutions while the predators stand for a collections of selection criteria. Initially, the entities are randomly populated in an emulating forest, which is characterized by a Moore-type two dimensional computing lattice. Each node in the lattice would, if not empty, accommodate at most one entity, either a prey or a predator [23]. A node has its neighborhood constituted by adjacent nodes in the lattice. The solving procedure starts with the initialization of the two populations. The prey are generated randomly in the context of a GA. Each predator is associated with a selection criterion, which is related to the objective function values of prey in its neighborhood as follows

$$
\Phi_{i j}=\omega_{j} F_{1, i}+\left(1-\omega_{j}\right) F_{2, i} ; \quad 0 \leq \omega_{j} \leq 1
$$

where $F_{1, i}$ and $F_{2, i}$ are values of the objective functions associated with a prey $i$ in the neighborhood of the predator $j$. The weight value, $\omega_{j}$, is uniquely generated for the predator $j$ through a uniform random number. During the solving process the predators prune the prey population based on the varying weighted fitness function; prey with low fitness value will be eliminated from the lattice. In the next generation new progenies of the surviving prey are generated through traditional GA operators (crossover and mutation) and are randomly located in the lattice. The predator activity on the basis of Eq. (1) continues from generation to generation. After prescribed number of generations, the surviving prey population is ranked according to the Fonseca criterion [24] and the best among them approximate the Pareto frontier.

The PPGA tool used in this study exhibits good stability and accuracy when performing numerical tests using a wide variety of standard test functions. In addition, it has been proved to be efficient for data-driven optimization problems as seen in many engineering applications, see e.g. [25] and therein references. For the sake of completeness, a list of GA parameters used with the PPGA tool is provided in Appendix.

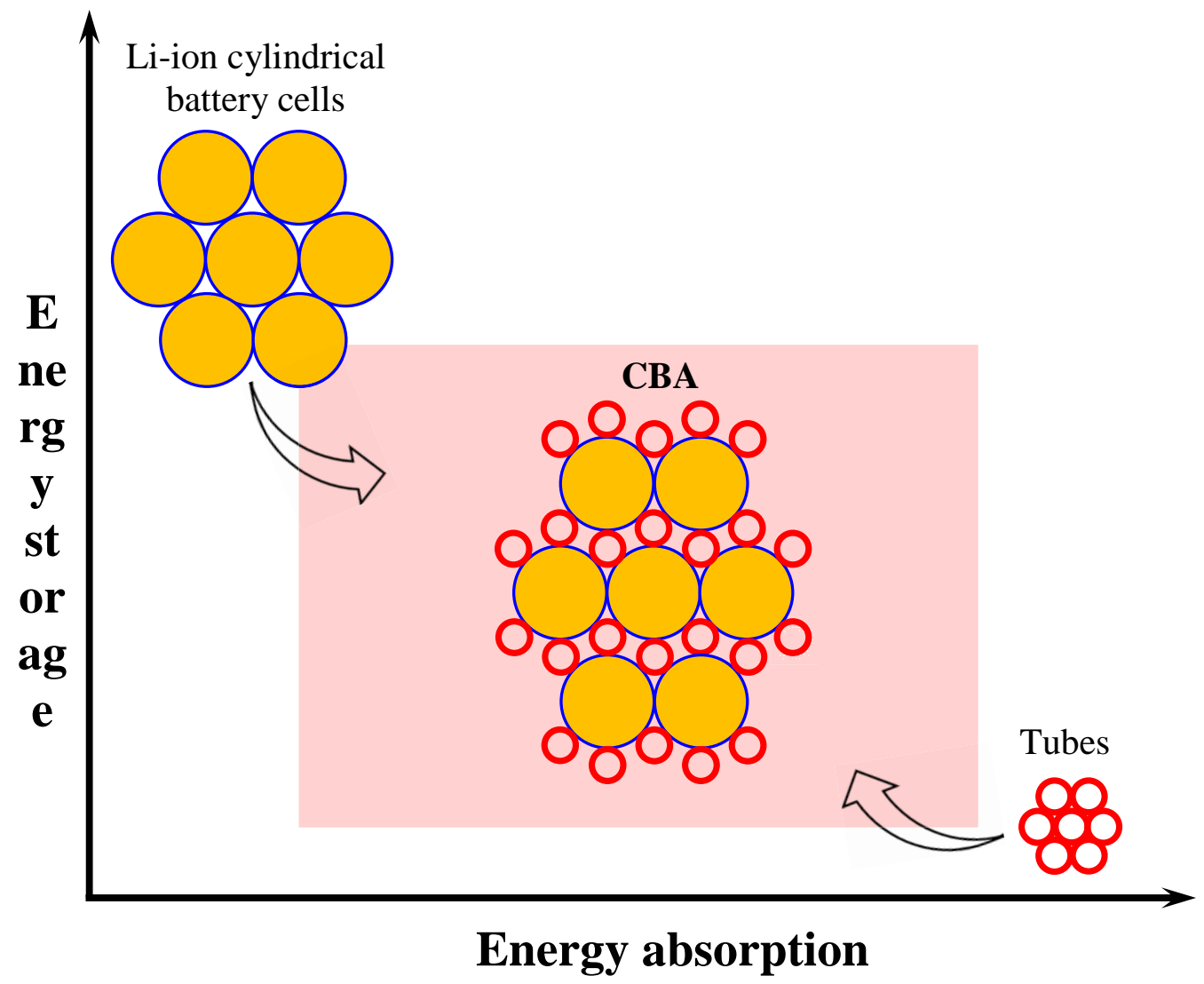


Figure 3. Design concept of CBA.

\section{Metamodeling technique}

The GA-based optimization technique employed in the optimization tool requires many evaluations of the objective and constraint functions. Therefore, the use of analytical expressions of those functions, if they exist, is preferred as the computation does not require extensive computation resources. Otherwise, with the absence of such relations, expensive numerical simulations using FE analysis tool are required. A reliance solely on FE simulations is computationally inefficient. As an alternative method for a function evaluation, the metamodeling technique to approximate the quantities of interest is applied [23, 25-27]. Metamodels are constructed using data generated by a limited number of FE simulations. This approach results in a significant reduction in computation effort and thus improves the performance of the optimization method. The strategy of using the metamodel technique has been proved to be efficient when a multi-objective optimization problem is solved by a population-based method, being either the PPDA [27-29] or any GA scheme [30, 31], which often requires a huge number of function evaluations.

The metamodels are constructed, trained and tested using FE simulation results of a group of selected configurations which are called sampling points. These statistically significant sampling points are generated through a design of experiment (DOE) process [32]. The Latin hypercube sampling (LHS) method [33] is adopted to achieve uniformity and balance of sampling points in the design window. The metamodels are constructed from the FE results at sampling points using an evolutionary neural network (EvoNN) approach [23, 25, 27]. Then, after being validated and tested, the metamodels are used to approximate quantities of interest. The significance of the EvoNN approach is its optimum trade-off between the complexity and accuracy of the models using the right amount of parameterization. Therefore, the obtained metamodels have no tendency of over- or under-fitting. Details of the method can be found in [23, 25]. Apparently, the metamodeling technique can effectively improve the performance of the proposed approach by reducing time of function evaluations. Associated by the construction of the metamodel, the PPGA can then effectively be applied to find the Pareto-front of given multi-objective optimization problem.

\section{Selection criterion}

After solving the multi-objective optimization problem, multiple Pareto solutions will be obtained. The configurations given by the Pareto set are equally valid optimal solutions. They suggest designers a list of feasible candidate solutions, thereby allowing for a fully informed choice of a final solution since the most satisfactory configuration is known a priori to be among members of the Pareto set. Yet, selection of the final configuration requires a criterion which regards the preference between the two objectives and/or other attributes. Any criterion that assists the selection of a final configuration is collectively considered a selection criterion.

\section{DESIGN OF CBA}

\subsection{Design constraints and requirements}

Being constructed from energy storage component CBA must fulfill certain design constraints, which are related to strict conditions of safety. That means the safety of CBA sets limits or constraints to the design space in which optimal solutions of the design problem are sought. The safety consideration of CBA involves failure of the energy storage constituent, which may fail electrically and/or mechanically under external loading. Therefore, appropriate experiments are required to determine such limits. These limits enter the design problem formulation in the form of design constraints. Outcome of the selection of components and their interface has a decisive influence on the design constraints as different energy storage constituents have different safety limits.

The design space is further narrowed down by the introduction of design requirements. Unlike design constraints which are strictly fulfilled, design requirements are related to desired features, specific characteristics and/or expectations of the design. Determination of the limits as for design requirements are subjective and pretty much application-dependent. In particular, CBA is aimed to be 
multifunctional. That is a design requirement indicating that CBA should be capable of being deformed and absorbing energy under external loading. Of course, CBA must satisfy the design constraints of safety. In order to be considered multifunctional the material system should be able to accommodate deformation greater than a pre-defined minimum level, which is set to be $\varepsilon_{\min }=5 \%$ in the present study. With this design requirement the energy absorption through plastic deformation of the energy absorbing constituents is made significantly possible. Such design requirement is introduced at the beginning of the design process to exclude bad solutions. Other design requirements such as cost and manufacturability may apply even though they are not considered in this study.

Note that both design constraints and design requirements will be collectively treated as constraints in the design problem formulation of CBA in later sections.

\subsection{Selection of components and their interface}

Only few options for the energy storage constituent satisfy the requirement of high energy storage capacity needs for EV applications. In particular, laptop battery cells 18650 are selected to be the energy storage component thanks to their superior energy density, availability and low cost [34, 35]. Data of a typical Panasonic battery cell, NCR18650A, is given in Table $\mathbf{1}$.

Options of the energy absorbing component are limited to cellular solids, foam materials and tube columns. Although axial crushing tube columns have high energy absorption capacity they are not preferred as sharp edges formed during crushing deformation may cause damage to batteries. Being lightweight and relatively high energy absorbing cellular solids such as honeycombs and foams are potential candidates. However, it is not efficient to use these materials in this specific space-filling application along with cylindrical battery cells because much work on shape preparation is required to guarantee compatibility between the two components. Radially collapsible tubes are selected to be the energy absorbing component of CBA after considering both energy absorbing capacity and conformability factors. The collapse mode of the tubes in radial direction is controllable and does not cause the sharp edges. Tubes made from aluminum alloy 6061-T6 are selected considering the factors of being lightweight, availability, and reasonable cost. The flow behavior of the material is characterized using a simplified relation as follows

$$
\sigma=A+B \bar{\varepsilon}_{p}^{n}
$$

where $A, B$ and $n$ are material constants; $\bar{\varepsilon}_{p}$ is the plastic strain. The material data of aluminum alloy 6061-T6 can be found in [36, 37] and are listed in Table 2. Moreover, aluminum sacrificing tubes can be used as an integrated cooling channel of the multifunctional battery material system. Thermal conductivity of the tube material is important as it strongly influences the temperature control of the system thereby could isolate heat up cells and prevent thermal run-away ignition from happening. Battery cells and aluminum tubes can touch and freely slide on each other during loading. Such direct contact encourages the heat exchange efficiency between the two constituents while facilitating the relative motion between the tubes and batteries.

Table 1: Characteristics of the Panasonic NCR18650A battery cell [34].

\begin{tabular}{|c|c|c|c|c|}
\hline $\begin{array}{c}\text { Nominal } \\
\text { voltage }(V)\end{array}$ & $\begin{array}{c}\text { Typical } \\
\text { capacity }(m A h)\end{array}$ & $\begin{array}{c}\text { Diameter } \\
(\mathrm{mm})\end{array}$ & $\begin{array}{c}\text { Length } \\
(\mathrm{mm})\end{array}$ & $\begin{array}{c}\text { Approx. } \\
\text { weight }(\mathrm{g})\end{array}$ \\
\hline 3.6 & 3070 & 18.50 & 65.30 & 47.5 \\
\hline
\end{tabular}

Table 2: Material data of aluminum alloy A6061-T6 tubes.

\begin{tabular}{|c|c|c|c|c|c|c|}
\hline $\begin{array}{c}\text { Young's } \\
\text { modulus }(\mathrm{GPa})\end{array}$ & $\begin{array}{c}\text { Poisson's } \\
\text { ratio }\end{array}$ & $\begin{array}{c}\text { Density } \\
\left(\mathrm{kg} / \mathrm{m}^{3}\right)\end{array}$ & $\begin{array}{c}\text { Yield stress } \\
(\mathrm{MPa})\end{array}$ & $\begin{array}{c}A \\
(\mathrm{MPa})\end{array}$ & $\begin{array}{c}B \\
(\mathrm{MPa})\end{array}$ & $n$ \\
\hline 68.9 & 0.3 & 2700 & 236 & 236 & 430 & 0.42 \\
\hline
\end{tabular}

\subsection{Selection of architecture}

To achieve a desired performance selection of architecture, or the arrangement of battery cells and sacrificing tubes, need to be carefully selected. Cylindrical battery cells and sacrificing tubes are desired to be assembled in such a way that the resulting system can efficiently accommodate and 
absorb a large fraction of kinetic energy imparted to the structure in a crash event whilst having a high stored energy density. Moreover, it can be proved that with cylindrical battery cells, the hexagonal packing arrangement (HPA) provides highest density as shown in Figure 4 (a). Cylindrical battery cells are represented by filled circles whose centers are arranged in a hexagonal lattice, and each circle is surrounded by six other circles. The packing ratio of this configuration is 0.91. That means a CBA with the HPA pattern can supply as much as $91 \%$ the energy of a parent battery of the same volume. Starting with the high energy density pattern, sacrificing tubes will then be inserted into the HPA configuration to protect the battery cells from excessive a predetermined loading limit and critical deformation caused by external loadings. According to studies of bi-model packing of circles $[38,39]$ there are several patterns for the arrangement of the tubes of radius $r$ and cylindrical battery cells of radius $R$. Note that CBA is intentionally designed to carry load only from frontal crash, i.e. the multifunctionality is available only in a preferred in a given direction. Therefore, tubes are preferably arranged to accommodate loading along the front-to-end direction of a vehicle and to maximize the performance of CBA in that direction. Such an assembly pattern is schematically depicted in Figure 4 (b) whereby close circles represents the battery cells and open circles are the sacrificing tubes. From this point onward a CBA implies the architecture derived from the densest energy storage configuration corresponding to the load carrying capability along vertical direction as shown in Figure 4 (b). It is worthwhile to note that only CBA configurations with identical sacrificing tubes (of the same dimensions and material) are investigated in this study.

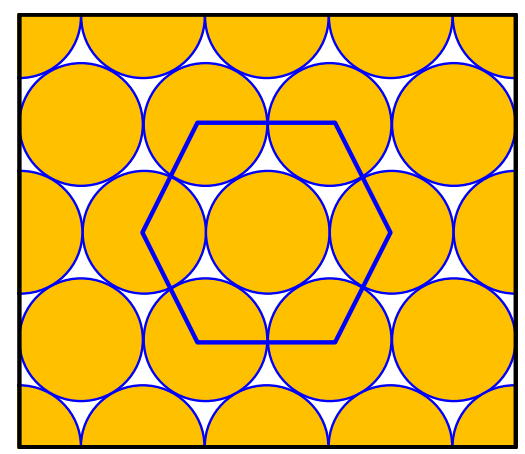

(a)

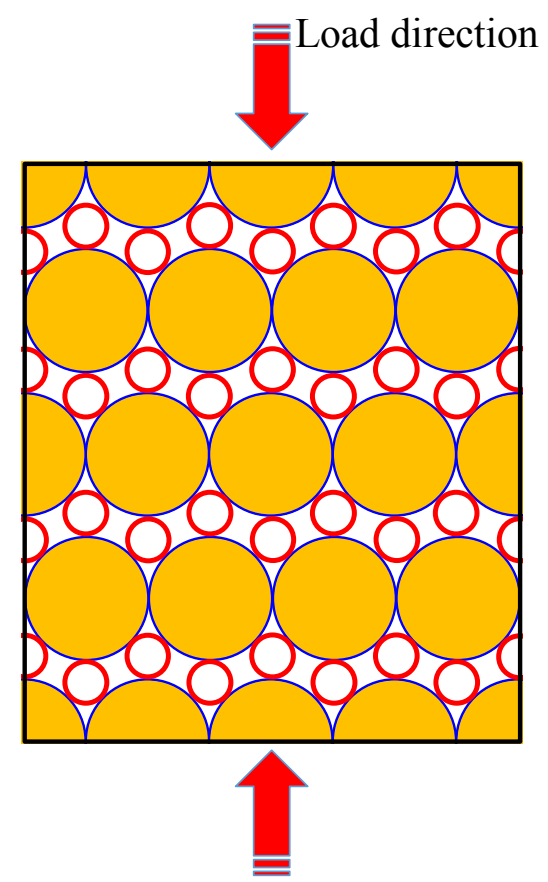

(b)

Figure 4. (a) Pattern with densest energy density of cylindrical batteries, and (b) The proposed architecture of CBA.

\subsection{Performance indices}

Characteristics of CBA are evaluated using a set of performance indices (PIs) [40, 41]. These PIs can be computed using analytical expressions or finite element (FE) simulations through the study of representative volume elements (RVEs). Cross-section views of the RVE are shown in Figure 5. The RVE of an undeformed configuration in Figure 5 (a) shows the basic structure of a CBA. The macroscopic structure of CBA can be generated by copying the RVE along two in-plane axes. An ideal deformed configuration is depicted in Figure 5 (b). With the introduction of the ideal deformed configuration, simplified calculations can be made to provide an initial estimation of quantities of interest. Refined results will then be obtained via FE simulations. 


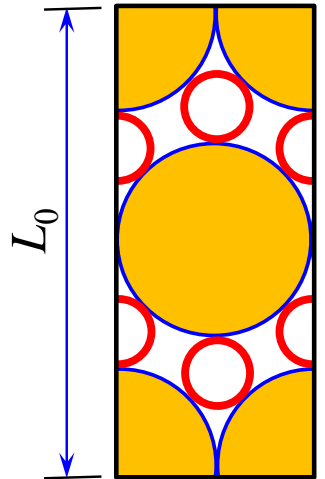

(a)

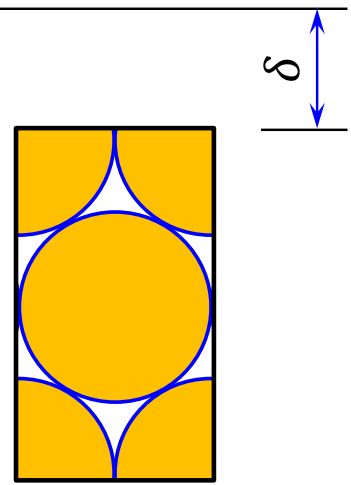

(b)

Figure 5. Representative element volume: (a) Undeformed configuration and (b) Ideal case of a fully collapsed configuration.

To assess the multi-functionality of a CBA, the following PIs are defined:

- Specific energy storage (SES) is a PI which represents the energy storage efficacy. SES defines the stored energy density of a CBA configuration by the stored energy-to-mass ratio as follows

$$
S E S=\frac{2 E_{\text {cell }}}{m_{\text {RVE }}}=\frac{2 E_{\text {cell }}}{4 m_{\text {tube }}+2 m_{\text {battery }}}
$$

where $E_{\text {cell }}$ and $m_{\text {battery }}$ are the storage energy and the mass of a NCR18650A battery cell obtained from Table 1. Note that there are 2 battery cells and 4 sacrificing tubes in the RVE. The mass of a tube is calculated by

$$
m_{\text {tube }}=\rho_{A l} \pi\left(2 r t-t^{2}\right) L
$$

where $\rho_{A l}$ is the density of the tube material (cf. Table 2), $r$ is the outer radius of the tubes, $t$ is the thickness of the tubes. The tube length, $L$, is equal to that of the battery cell.

Maximizing SES aims at a lighter system of the same energy capacity. Weight saving and driving range enhancement objectives can be achieved with a CBA configuration of high specific energy. The variation in specific energy storage versus battery radius and thickness of CBA according to equation (3) is plotted in Figure 6 (a).

- Specific Energy Absorption (SEA) determines the crush resistance efficacy of a CBA configuration by the absorbed energy-to-mass ratio when the system is subjected to a compressive crushing load. The crush resistance capability of CBA is an outcome of the tube collapse. SEA is calculated from the area under the crushing force-displacement curve by

$$
S E A=\frac{1}{m_{R V E}} \int_{0}^{\delta_{\text {iim }}} F d \delta
$$

where $F$ is the crushing force and $\delta_{\lim }$ is the limit crush length corresponding to the maximum allowed displacement of CBA without violating the design constraints. CBA with high value of $S E A$ is more favorable as it partly contributes to the battery and occupant protection.

- Mass density $\left(P I_{M}\right)$ is calculated by the ratio of the overall mass, $m_{R V E}$, and volume, $V_{R V E}$, of the RVE:

$$
P I_{M}=\frac{m_{R V E}}{V_{R V E}}=\frac{4 m_{\text {tube }}+2 m_{\text {battery }}}{4 R L\left(R+r+\sqrt{r^{2}+2 R r}\right)}
$$

$P I_{M}$ is used to compare the weight saving of different configurations. It can also be applied as a design criterion as a low mass density CBA is always desired: between two configurations of CBA of similar features, the one with lower values of $P I_{M}$ is preferred. Following Figure 6 (b), the lowest values and most desirable value of $P I_{M}$ are obtained for tubes with large radius and thin walls. 
- Battery volume fraction $\left(P I_{B}\right)$ is the ratio of the total volume of batteries within the RVE to that of the RVE. $P I_{B}$ can be used to compare the stored energy capacity of different configurations. For a CBA configuration the battery volume fraction is calculated by

$$
P I_{B}=\frac{\pi}{2} \frac{R}{R+r+\sqrt{r^{2}+2 R r}}
$$

The packing ratio increases with decreasing tube radius and is independent of tube thickness as plotted in Figure 6 (c).

- Ideal densification strain $\left(P I_{\varepsilon}\right)$ is the maximum strain that a CBA can achieve. The ideal densification strain is calculated from the undeformed and ideal deformed configuration of the RVE when batteries of neighboring rows come to touch each other, cf. Figure 5,

$$
P I_{\varepsilon}=\varepsilon_{D}=\frac{\delta}{L_{0}}=\frac{\left(R+r+\sqrt{r^{2}+2 R r}\right)-\sqrt{3} R}{\left(R+r+\sqrt{r^{2}+2 R r}\right)}
$$

Note that in most of the cases an ideal densification cannot be reached due to violation of the safety of batteries, i.e. force acting on battery exceeds the failure load before the ideal collapsed configuration is reached. However, this PI can possibly be used to eliminate bad configurations which do not have the capability to accommodate deformation as specified in the design requirements. Figure 6 (d) shows the dependence of the ideal densification on tube thickness and radius.

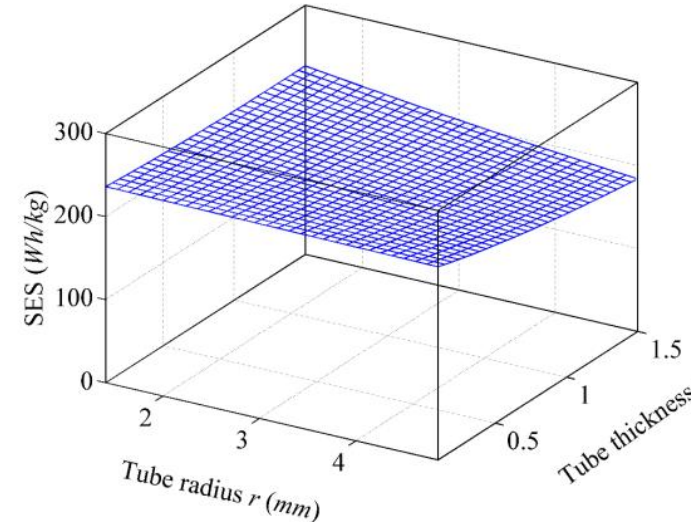

(a)

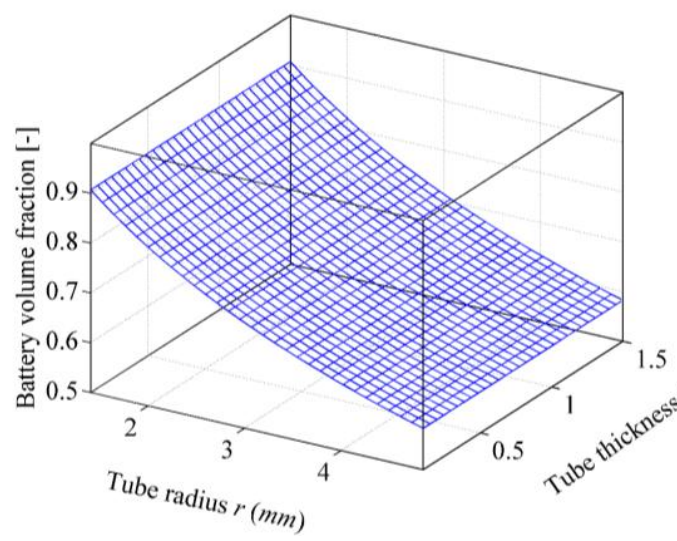

(c)

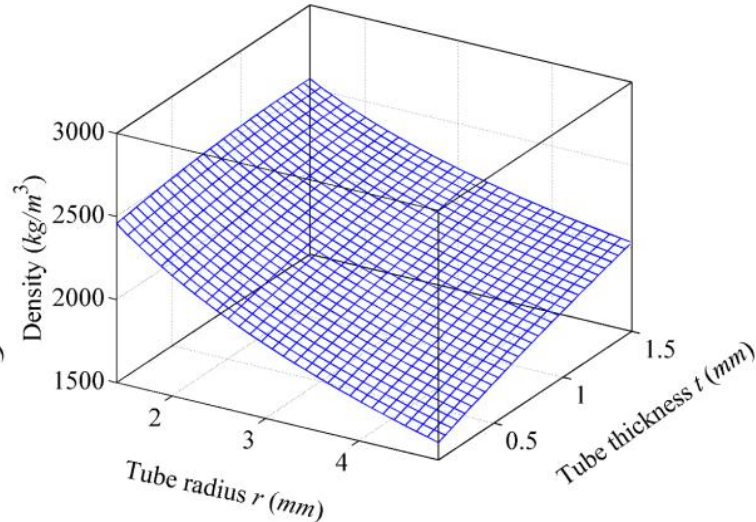

(b)

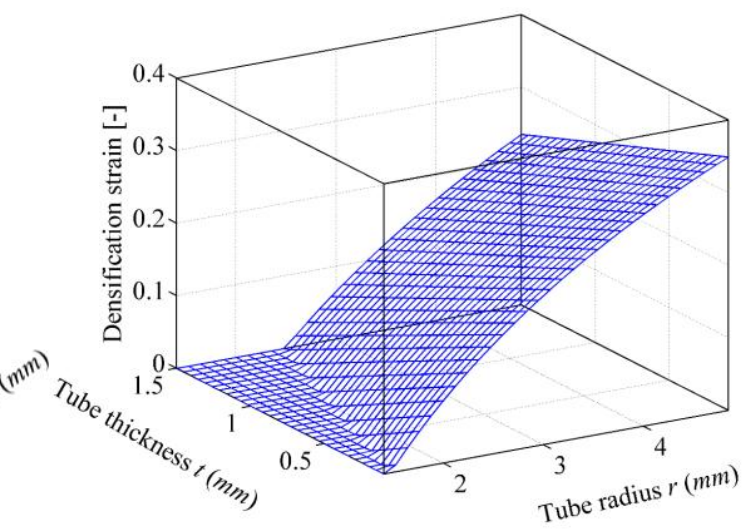

(d)

Figure 6. Variation of (a) the Specific Energy Storage (SES) performance index, (b) the mass density performance index, (c) the battery volume fraction performance index, and (d) the ideal densification strain performance index in the design parameter space. 
- Initial collapse load $\left(P I_{C}\right)$ is the minimum crushing force amplitude that may cause a single tube collapsed under a similar loading configuration to that in the CBA architecture. A representation of the loading condition of a single tube in the proposed topology of CBA is depicted in Figure 7 (a). A further simplified model is shown in Figure 7 (b). By adopting an analytical solution for initial collapse load of tube supported in a V-block, as shown in Figure 7 (b), the initial collapse load of tubes is calculated by [42]:

$$
P I_{C}=F_{C}=\frac{4 M_{P}}{r_{m}} \cot \left(\frac{\pi+\alpha}{8}\right)=\frac{k \sigma_{P} t^{2}}{r-0.5 t} \cot \left(\frac{\pi+\alpha}{8}\right)
$$

where $M_{P}$ is the fully plastic moment per unit length, $r_{m}$ is the mean radius of the tube, $k=2 / \sqrt{3}$ is the factor accounting for the plane strain condition, $\sigma_{P}$ is the yield strength of the tube material, $\alpha$ is the angle between the two inclined surfaces as depicted in Figure 7 (b).

The initial collapse load indicates how early a CBA configuration can be switched to energy absorbing mode. If the tubes passes a low $F_{C}$ then the energy absorption calculated by the area under the force-displacement curve can be small. However, if tubes have a collapse load that is too high then batteries may fail at a load lower than the value of $F_{C}$. Thus, this index is used to eliminate bad configurations that do not meet the battery safety requirement or possibly provide low capability of energy absorption.

- $\quad$ Nominal plateau stress, $\sigma_{P}$, is calculated by [43]

$$
P I_{\sigma}=\sigma_{P}=\frac{S E A_{v o l}}{\varepsilon_{\lim }}
$$

where $S E A_{\text {vol }}$ is the energy absorption per unit volume; $\varepsilon_{\text {lim }}$ is the limit strain above which the battery safety is not guaranteed. This $P I$ will be used for comparison the energy absorption capability of CBA with that of some absorbing materials.

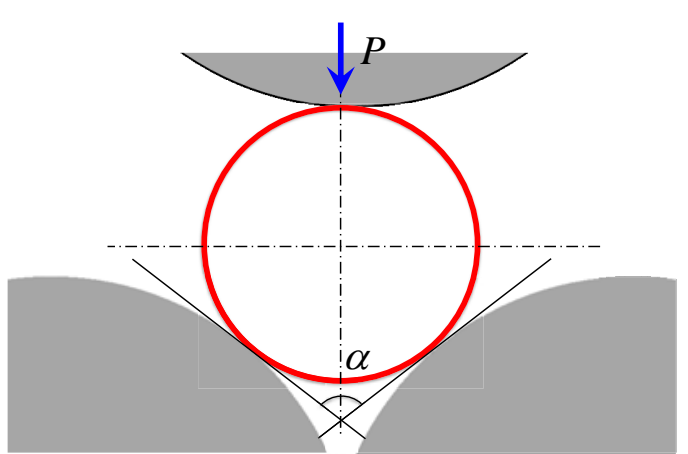

(a)

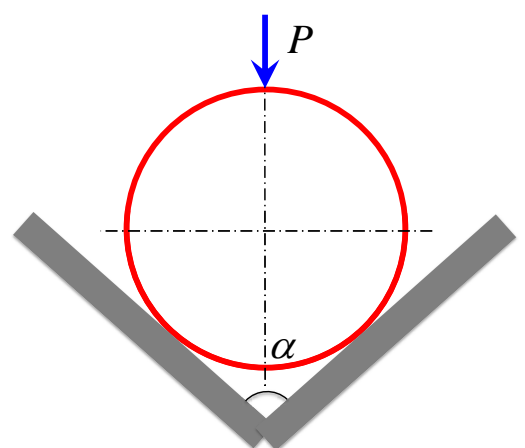

(b)

Figure 7. (a) Loading condition in a CBA configuration and (b) its simplified model.

Note that calculation of some PIs cannot be made analytically due to difficulty in obtaining explicit expressions, or error from using over-simplified expressions. In particular, the ideal deformed configuration Figure 5 (b) can be unrealistic in certain cases because the geometry of the tubes after being collapsed are not accounted for. Calculations based on the ideal deformed configuration can be misleading. Therefore, FE simulations are employed to test the validity of the analytical calculations. Then, FE results are also used for construction of metamodels to approximate the quantities of interest. FE simulations of the RVE are conducted using the commercial software ABAQUS [44] as described in Appendix A.1.

\section{THE OPTIMIZATION PROBLEM}

Optimal configurations of the multifunctional battery system CBA are sought through solving a multi-objective optimization problem of two conflicting objectives which aim at maximizing the SES and $S E A$, at the same time. To formulate the optimization problem, choice of design variables and 
introduction of constraints are presented first. Then, the objective functions are defined in this section.

Radius and thickness of the tubes, i.e. $r$ and $t$, are selected as design variables to be determined in the optimization procedure leading to the Pareto optimal configurations of CBA: $\mathbf{x}_{\text {opt }}=\left(r_{\text {opt }}, t_{\text {opt }}\right)$. Constraint functions define limits that are not allowed to be violated. The constraints are also derived from the design constraints and requirements and are functions of the design variables.

In a simplest form, the constraint functions define lower and upper bounds of the design variables $\left(x_{j}^{L} \leq x_{j} \leq x_{j}^{U}, j=1,2\right)$. Provided that radius and thickness of tubes must be constrained to make the assembly possible, large radius tubes cannot be employed as they invade spaces for neighboring tubes. Also, small radius tubes are trapped in the empty spaces formed by three adjacent battery cells and therefore have no contribution to the energy absorption. Likewise, a thick tube corresponds to a high collapse load which does not contribute to the protection of batteries. On the other end, collapse of very thin tubes is easy but its contribution to the SEA is small and thus is not desired. Based on these considerations, geometrical constraints are introduced in the form of equations (11) and (12).

$$
\begin{aligned}
& 1.4078<r<4.8530 \\
& 0.1<t<1.5
\end{aligned}
$$

Together with the condition of $\varepsilon_{\min }=5 \%$, these constraints define a window in the design parameter space over which sampling points are populated. The imposed constraints serve as a filter to remove bad configurations as early as from the step of sampling point generation. This improves the quality of the information obtained from FE simulations in the following step as they are confined in a domain of interest rather than being scattered and containing useless data. Even though bad combinations of the design parameters can be discarded by the optimization process, it is always more economical to narrow down the search space as much as possible.

Being an essential design constraint, the safety of batteries must be guaranteed in any optimal considerations. Therefore, CBA should be designed to function within a safety limit of the batteries, in both electrical and mechanical aspects. To fulfill this requirement, the following actions are required. First, a failure limit of the battery should be identified. Second, in all steps of the design procedure, this limit must not be exceeded.

Experiment setup as shown in Figure 8 (a) is used to determine the failure load of the batteries. The failure load, $F_{\text {failure }}$, is determined by considering both mechanical and electrical modes. Figure 8 (b) shows that the electrical failure mode corresponds to abrupt voltage drops while mechanical failures are signified by loss of load carrying capability. It is seen that batteries fail in the electrical failure mode rather than the mechanical one. The failure load of the batteries is determined at the point of voltage drop $F_{\text {failure }} \approx 25 \mathrm{kN}$. Then, the limit load per unit length to be used in the plane strain RVE analysis is calculated by

$$
F_{\lim }=\frac{F_{\text {failure }}}{L}
$$

where $L=65.3 \mathrm{~mm}$ is the length of batteries. This gives $F_{\lim }=192 \mathrm{~N} / \mathrm{mm}$. Note that the limit strain $\varepsilon_{\lim }$ in (10) is determined to correspond the $F_{\lim }$ condition. Then, protection of batteries through the collapse of sacrificing tubes is possible when the initial collapse load of tubes is lower than the failure load of battery. For plane strain condition, by combining (9) and (13), the constraint becomes

$$
g_{1}(\mathbf{x})=F_{C}=\frac{4 k \sigma_{P} t^{2}}{r-0.5 t} \cot \left(\frac{\pi+\alpha}{8}\right)<F_{\lim }
$$

Furthermore, to meet the design requirement defined in section 3.1 the ideal densification strain must be greater than a chosen strain level of $5 \%$, i.e.

$$
g_{2}(\mathbf{x})=\varepsilon_{D} \geq \varepsilon_{\min }=0.05
$$


Two objective functions, namely $f_{1}(\mathbf{x})$ and $f_{2}(\mathbf{x})$, are defined to measure the specific energy storage and the specific energy absorption, both of which are then maximized:

$$
\begin{aligned}
& f_{1}(\mathbf{x})=\text { Specific energy storage }(S E S) \rightarrow \max \\
& f_{2}(\mathbf{x})=\text { Specific energy absorbtion }(S E A) \rightarrow \text { max }
\end{aligned}
$$

Along with the constraints given by (11), (12), (14), and (15), equations (16) and (17) define a biobjective Pareto-optimal problem. Increase in energy storage and impact and reduction in weight to improve the performance of the battery system are contradicting objectives. The multi-objective optimal design problem is solved using the method presented in section 2.

\section{RESULTS AND DISCUSSION}

Provided that CBA should be capable of accommodating a minimum deformation of 5\% as a design requirement, the design window of $\mathrm{CBA}$ is described in Figure 9. In the design window the constraints (11), (12), (14), and (15) are satisfied. However, the design window does not guarantee the safety condition of battery, i.e. the load acting on batteries may exceed the battery failure load at an actual strain value lower than 5\% regardless (15). Despite its limitation, the design window obtained in Figure 9 via simplified calculations can be used to eliminate bad configurations in the step of generating sampling points. A refined design window will later be obtained from results of FE simulations at sampling points.

The crushing response of CBA is investigated in the quasi-static regime using FE simulations for a number of configurations. Selection of these sampling points follows the LHS method (cf. section 2). Figure 10 (a) shows a typical load-displacement curve of CBA. Like other cellular materials, the response of CBA consists of 3 regimes: an elastic response followed by a plateau regime and eventually a densification regime of sharply rising stress. It can be seen that the calculated initial collapse load, $F_{C}$, using (9) can reasonably predict the force at which sacrificing tubes starts to collapse. The failure load $F_{\text {lim }}$ is also shown to indicate a limit below which the battery safety is guaranteed.

CBAs of different configurations respond differently to the crush loading. The load level in the plateau regime may increase rapidly due mainly to geometrical constraining effect prior to densification. This is related to interaction between tubes which introduces more complex behaviors such as appearing of multiple collapse points, up-shifting of multiple plateau levels, and triggering earlier confinement. The load-displacement curve of another configuration with different values of tube radius and thickness is shown in Figure $\mathbf{1 0}$ (b). The curve exhibits two plateau regions before reaching the failure load $F_{\text {lim }}$. The behavior is related to different deformation stages of sacrificing tubes as depicted in Figure 11. In the loading process, starting from an undeformed configuration, tubes experience initial collapse, tube-to-tube impingement, second collapse and densification. Impingement of tubes triggers the second collapse with the formation of new plastic hinges, thereby promoting further energy dissipation. That explains the formation of multiple plateau regions in Figure 10 (b) rather than a single one in Figure 10 (a).

A metamodel is constructed following the method given in section 2 to approximate SEA. As shown in Figure 10 the failure load of batteries, $F_{\text {lim }}=192 \mathrm{~N} / \mathrm{mm}$, is reached before the densification strain can be achieved. Therefore, the SEA is computed by the area under the load-displacement curve up to the point where failure load is reached. The limit crush length, $\delta_{\lim }$, corresponding to the failure load is also determined. The shaded area under the curves in Figure $\mathbf{1 0}$ represents the computed $S E A$. The battery safety consideration is incorporated when calculating $S E A$ from the shaded areas. Note that the calculation does not account for elastic recovery. Construction of a metamodel from the FE simulation results at sampling points is presented in Appendix A.2.

Using simulation results of sampling points, a metamodel is constructed to approximate the limit crush length $\delta_{\lim }$. Then, the limit strain that a CBA can achieve before batteries fail is calculated by replacing $\delta=\delta_{\lim }$ in (8) 


$$
\varepsilon_{\lim }=\frac{\delta_{\lim }}{L_{0}}=\frac{\delta_{\lim }}{\left(R+r+\sqrt{r^{2}+2 R r}\right)}
$$

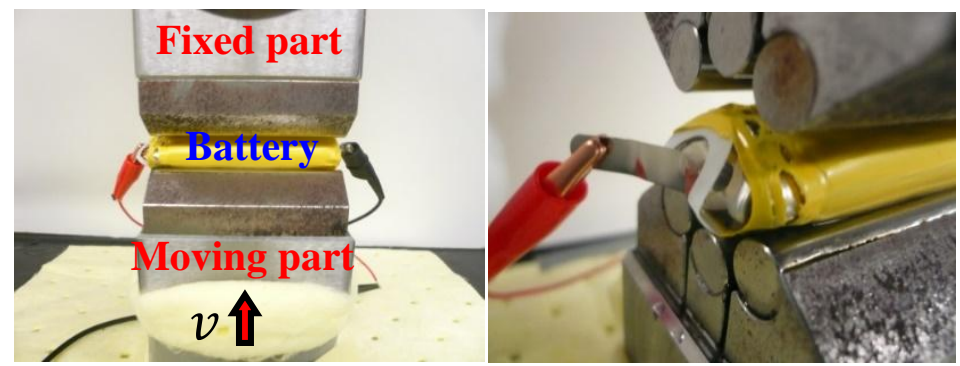

(a)

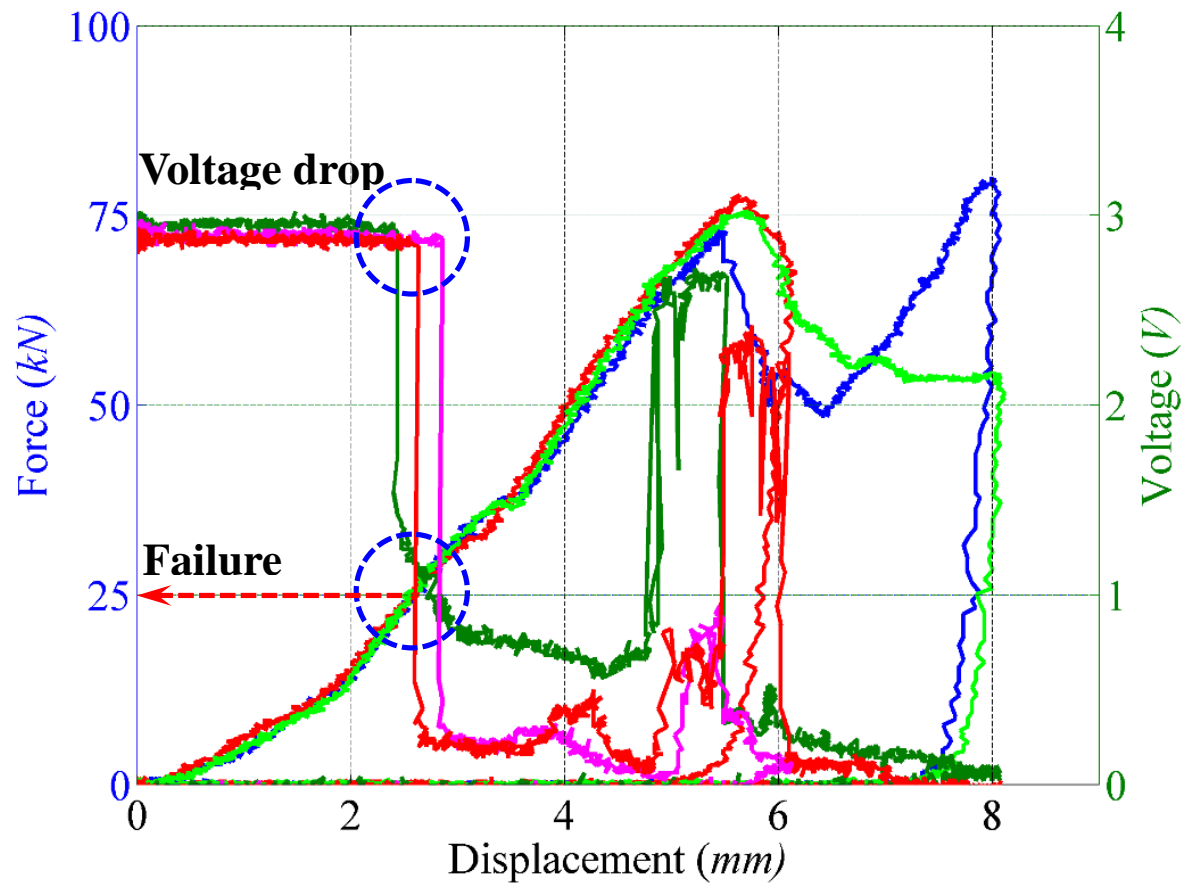

(b)

Figure 8. (a) Experiment setup, (b) Determination of the failure load by considering both mechanical and electrical failure modes.

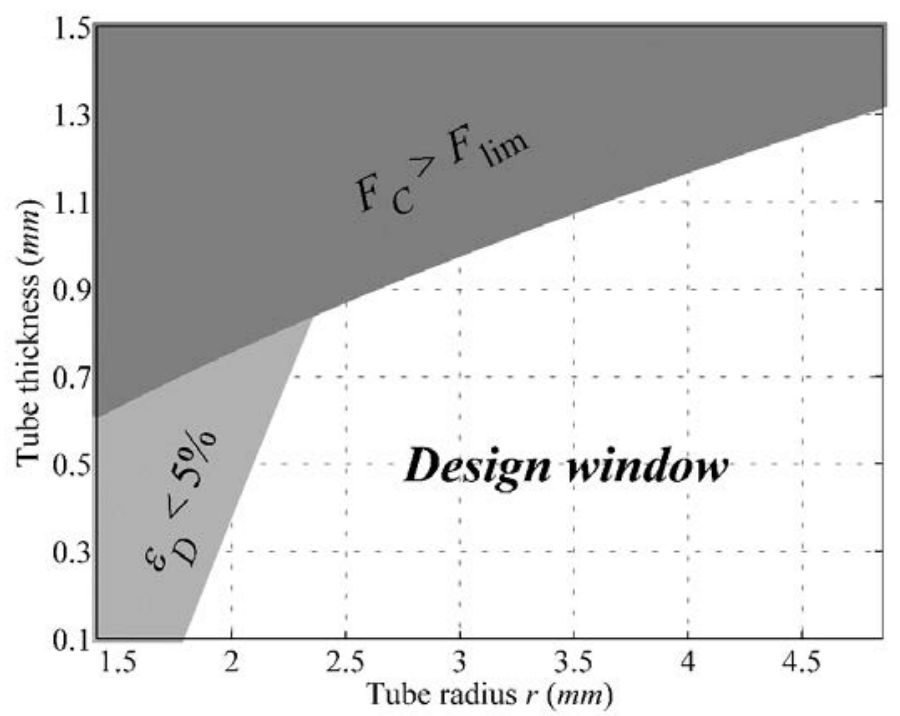


Figure 9. Constraints in the design variable space to define a design window.

To reflect the fact that battery must still be within the safety limit when CBA deforms and absorbs crush energy, the initial design constraints $\varepsilon_{D}>5 \%$ is replaced by $\varepsilon_{\text {lim }}>5 \%$. The updated constraint is applied to guarantee that CBA can be actually deformed at least 5\% strain without failing batteries. As the condition with allowed actual deformation is more restricted than that with the ideal one, the former introduces a tighter constraint. With this update the design window shrinks significantly as shown in Figure 12. Boundary of the design window corresponds to $\varepsilon_{\lim }=5 \%$. It can be seen that the condition $\varepsilon_{\lim }>5 \%$ includes the constraints in (11), (12), (14) and (15).

A refined metamodel is constructed to better approximate $S E A$ of configurations in the updated design window. Unlike the first built metamodel which uses FEA results at all sampling points, the refined metamodel employs only ones that fulfill the condition $\varepsilon_{\text {lim }}>5 \%$. This helps to better capture variation of SEA in a more confined space of the design window. Additional sampling points are selectively augmented, thereby improving the predictive capability of the refined metamodel.

FE simulation results of the sampling points allow for an understanding of how the design parameters and objectives are correlated. The 2D parallel coordinate plot in Figure 13 presents the multiple interactions among the quantities. Vertical axes of the plot measure the variation of tube radius, thickness, SEA and SES. Inputs and outputs of any sampling points are normalized and represented by points on corresponding vertical axes. Connecting lines sharing common points at an axis belong to the same CBA configuration. The displayed levels on the left vertical axis are normalized values of the qualities. The normalization is made by dividing all member of each group by the corresponding maximum value of that category. The dataset contains only admissible configurations which are located in the feasible region in the design space. The variation of SES is small compared to that of SEA.

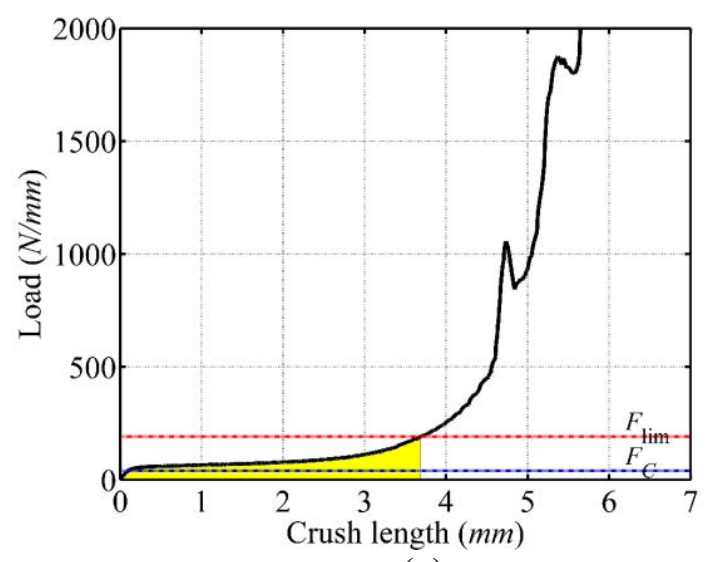

(a)

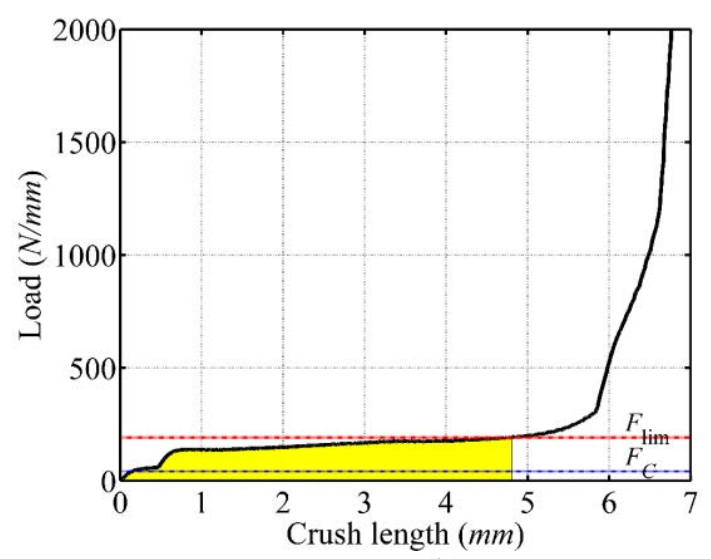

(b)

Figure 10. Load-crush length curve with (a) one plateau region, and (b) two plateau regions.

Figure 13 differentiates three groups: group 1 of highest SES (in blue color), group 2 of highest $S E A$ (in red color), and group 3 of other configurations (in grey color). None of the samples achieve maximum $S E S$ and $S E A$ simultaneously. Group 1 has low $S E A$ values which are populated to the bottom of the $S E A$ axis. Blue lines show that any improvement in $S E A$ results in a degradation in $S E S$. Similarly, group 2 has intermediate values of $S E S$, though not the lowest but significantly lower than the values of group 1. An increase in SES of this groups leads to a decrease in SEA. Note that the samples are randomly generated and do not necessarily belong to the Pareto front. Still, this observation implies that SES and SEA are competing: an increase in SES is achieved at the expense of $S E A$ and vice versa. Therefore, optimal conditions of the system design variable are trade-off solutions of the two conflicting objectives. Moreover, configurations of highest SES value correspond to small and thin tube while samples of high SEA are of larger and thicker tubes. The 
observation indicates that competing modifications of the design parameters are required to meet the both performance objectives.

The energy absorbing capability of CBA is then compared with that of other materials whose data is available in the literature, e.g. [43]. Figure 14 shows the correlation between SEA and nominal plateau stress (cf. equation (10)) of CBA and various cellular materials. Figure 14 (a) and (b) show not only comparison of SEA but also that of mass density and storage energy capacity. In Figure 14 (a) the mass density of CBA is compared with that of foam materials. Numbers in the plot indicates the mass density of different foams. A color-coded presentation of the mass density of CBA is also provided. The color bar shows value ranges corresponding to the displayed colors. Figure 14 (b) displays a spectrum of the storage energy capability for the foams and CBA. The color bar reads as the storage energy level of the materials in terms of volume fraction of embedded battery.
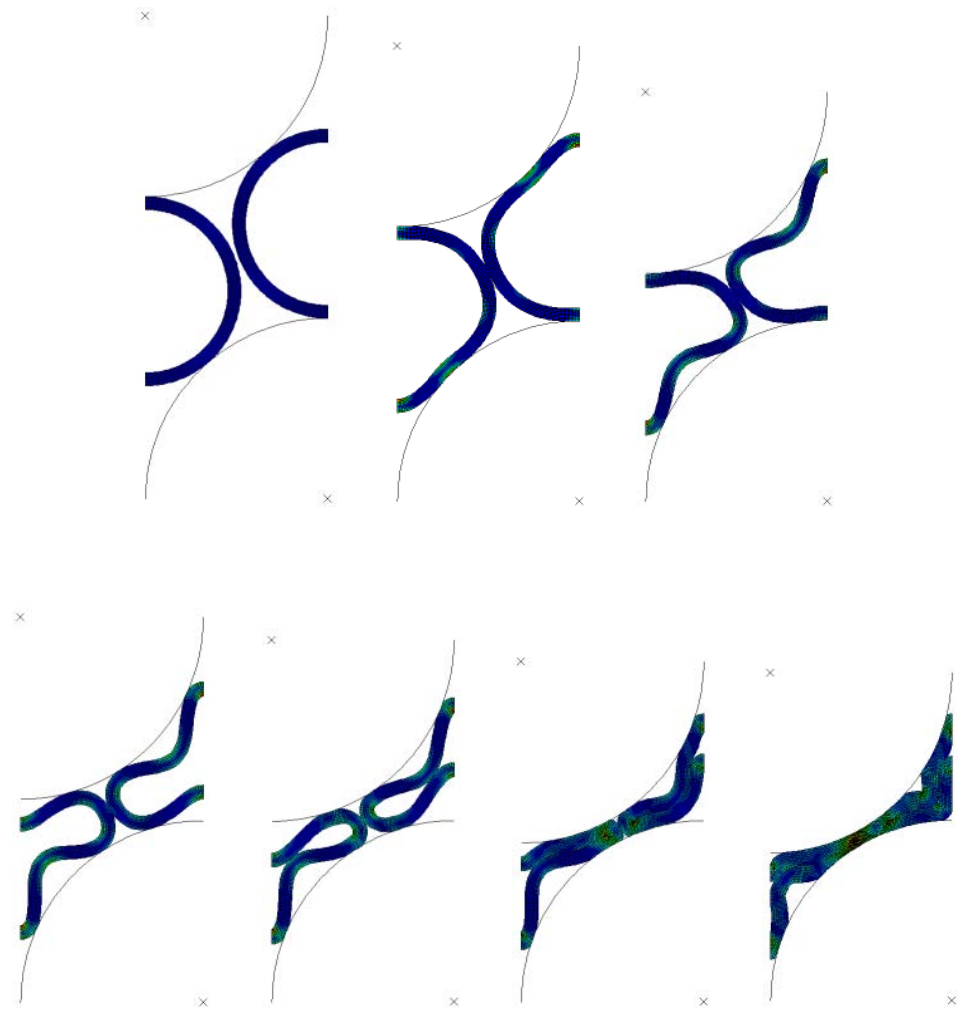

Figure 11. Sequence of deformed states of tube crushing in CBA, FE simulation with RVE.

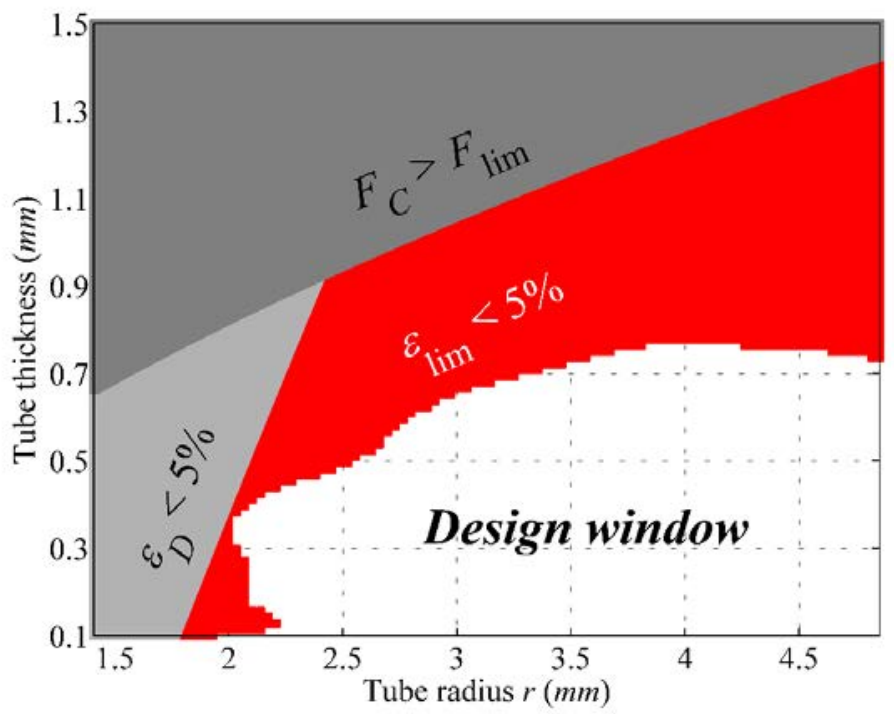

Figure 12. Updated design window. 


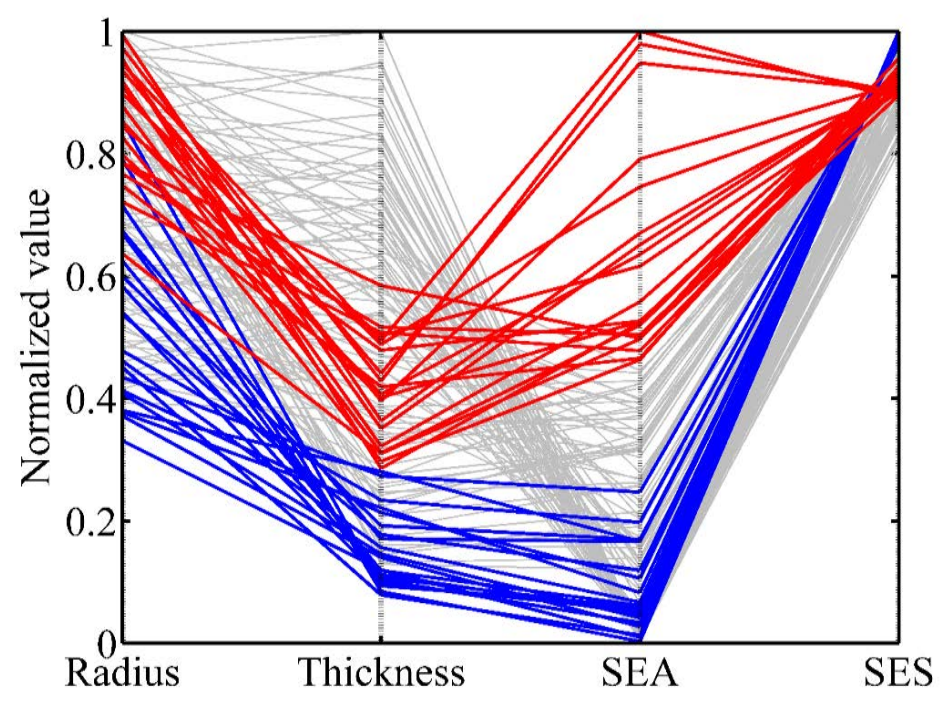

Figure 13. Parallel coordinate plot showing correlation between design parameters and objectives for sampling points in 3 groups: high SES (blue), high SEA (red), and the remaining (grey).

The plots show that SEA data of CBA resides off and lower to the right of the diagonal band formed by aluminum foams. The plateau stress of CBA is in the upper-mid range of the chart reflecting the fact that the maximum allowed strain is not dictated. CBA exhibits inferior $S E A$ to those of high-density aluminum foam materials, cf. Figure 14 (a). However, the energy absorbing performance of CBA is quite comparable to foams of low to mid density. Note that the energy absorption level of CBA is limited by the failure load of the battery. Therefore, one way to improve the $S E A$ of CBA is to bring up the magnitude of $F_{\text {lim }}$ as high as possible. This suggests a new design criterion of battery construction.

Due to the heavy weight of battery cells embedded in CBA, the mass density of CBA is one order of magnitude higher than that of mid-density aluminum foams. However, the multifunctional material system CBA defeats all the absorbing materials in terms of energy storage capability, cf. Figure 14 (b). Given that $\varepsilon_{\lim } \geq 5 \%$, the battery volume fraction of a CBA ranges from about $58 \%$ to above $80 \%$. As foam materials do not contain any energy storage unit, the battery volume fraction for these materials is zero. It is worthwhile to recall that if only battery cells are packed, a maximum battery volume fraction of $91 \%$ is achieved.

For the sake of completeness, information of the battery constituent should be represented in Figure 14. However, unlike other systems, such data is not available due to the absence of the energy absorption mechanism in battery cells alone. With the assumption of being rigid in FE simulations, batteries have a zero energy absorption $(S E A=0)$ and infinite plateau stress $\left(\sigma_{P} \rightarrow \infty\right)$. The value of the energy storage volume fraction of a single battery cell is $100 \%$. However, an assembly of multiple cells using the densest packing configuration gives a maximum energy storage volume fraction of $91 \%$. The corresponding mass density of the assembly is $P I_{M} \approx 2.6 \mathrm{Mg} / \mathrm{m}^{3}$ while it is for a single cell $P I_{M} \approx 2.9 \mathrm{Mg} / \mathrm{m}^{3}$.

The genetic algorithm approach in section 2 is then applied to solve the formulated bi-objective optimization problem. A Pareto frontier displaying the entire set of optimal solutions in the design objective space is plotted in Figure 15. The Pareto frontier determines a boundary between feasible and infeasible regions in the objective space $f_{1}-f_{2}$. That means no configuration in the design window can reach the region above the Pareto frontier. Good trade-off between the two objectives can also be visualized from Figure 15. The Pareto frontier exhibits a "knee-like" point across which $S E S$ experiences a significant change when $S E A$ slightly varies.

Solutions obtained from the Pareto optimal set are then plotted in the design variable space in Figure 16. Color-coded symbols represent the magnitude of the two objective: Figure 16 (a) 
displays the level of SEA while Figure 16 (b) shows the level of SES corresponding to each Pareto solution. The optimal solutions are populated and grouped in clusters and some scattered points. Particularly, optimal tube thickness is distributed in a confined range from $0.1 \mathrm{~mm}$ to $0.6 \mathrm{~mm}$ beyond which either the design constraint is violated or CBA becomes sub-optimal. On the other hand, optimal values of the tube radius span in a wide range, though not continuous. Configurations of low thickness and low radius have lowest $S E A$ and highest $S E S$ while solutions with high thickness and high radius have highest $S E A$ and lowest $S E S$. Intermediate magnitudes of $S E A$ and $S E S$ are achieved by combination of mid-range values of thickness and radius. Blank areas imply combinations of radius and thickness values in those zones lead to either sub-optimal CBA or violation of the design constraints.

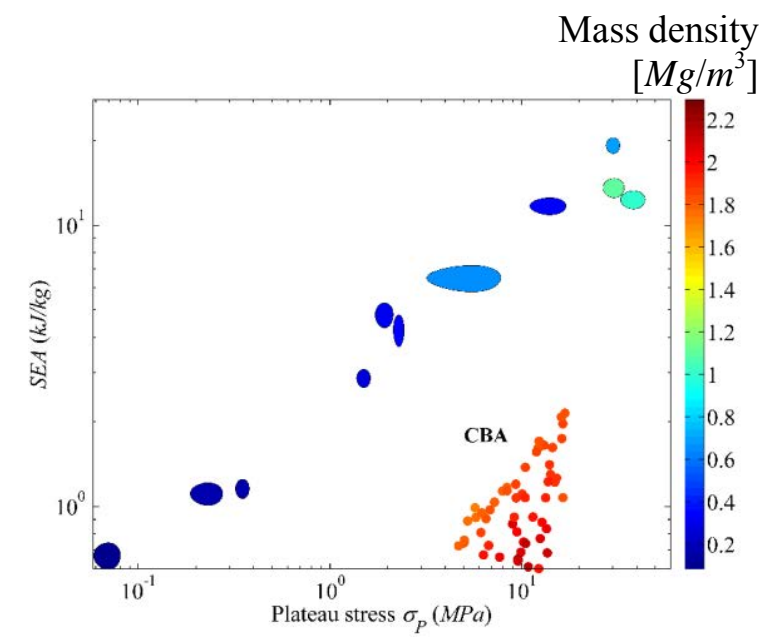

(a)

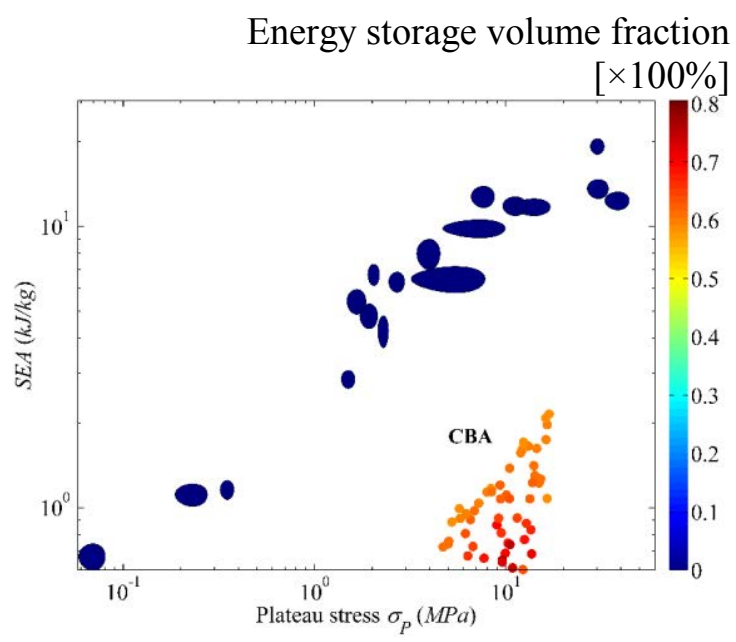

(b)

Figure 14. Comparison of the specific energy absorption and plateau stress among various materials. The color bar shows variation of (a) mass density, and (b) battery volume fraction of CBA. Numbers in (a) indicate mass density of foam materials.

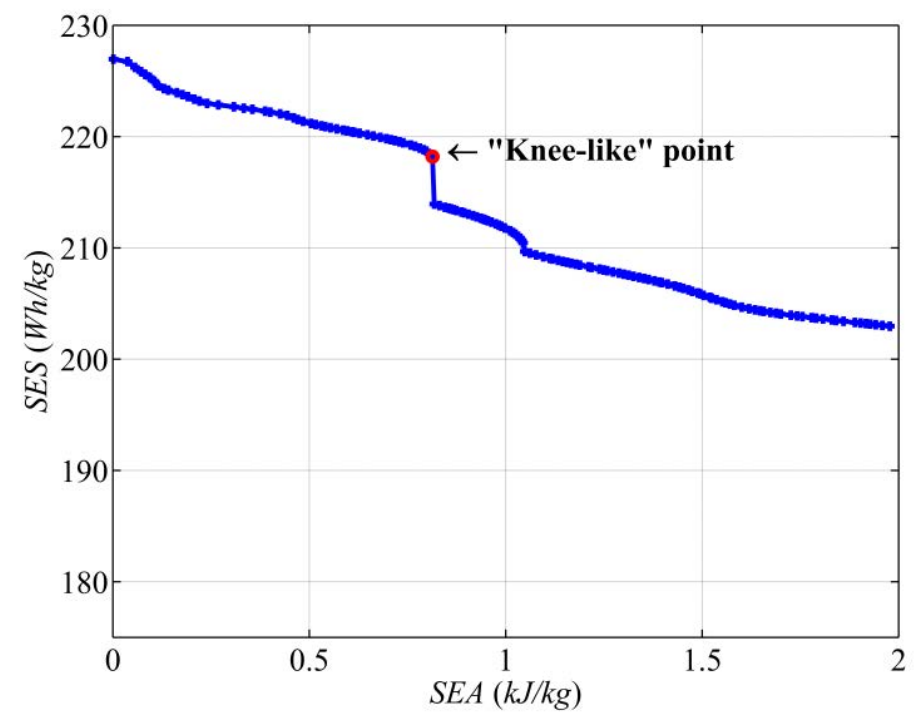

Figure 15. Pareto frontier of the bi-objective optimization problem (with $0.02 \mathrm{~kJ} / \mathrm{kg} \leq S E A \leq 1.98 \mathrm{~kJ} / \mathrm{kg}$ and $203 \mathrm{Wh} / \mathrm{kg} \leq S E A \leq 227 \mathrm{Wh} / \mathrm{kg}$ ).

Selection of a final configuration requires a selection criterion regarding the preference between the two objectives and/or further factors. For the current problem, to this point, choice of the final tube dimensions remains open but is subjective to which criterion, between the storage energy and absorption energy, is more important. The selection criterion can be material availability, 
manufacturing cost and/or other performance considerations. To address a preferable mechanical response of CBA under crush loading, a final configuration is aimed to reduce the load level on batteries and to be able of accommodating a high deformation level. Therefore, the performance indices $F_{C}$ and $\varepsilon_{\lim }$, which are used as constraints initially, are then re-casted to be selection criteria.

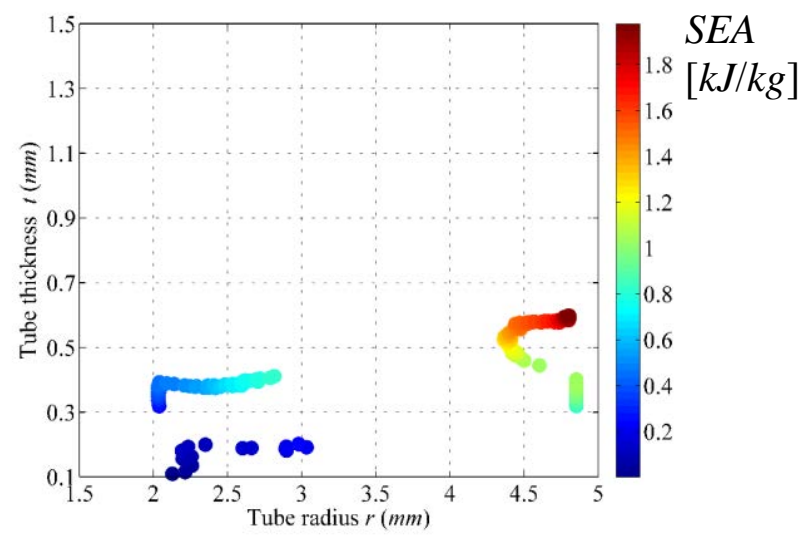

(a)

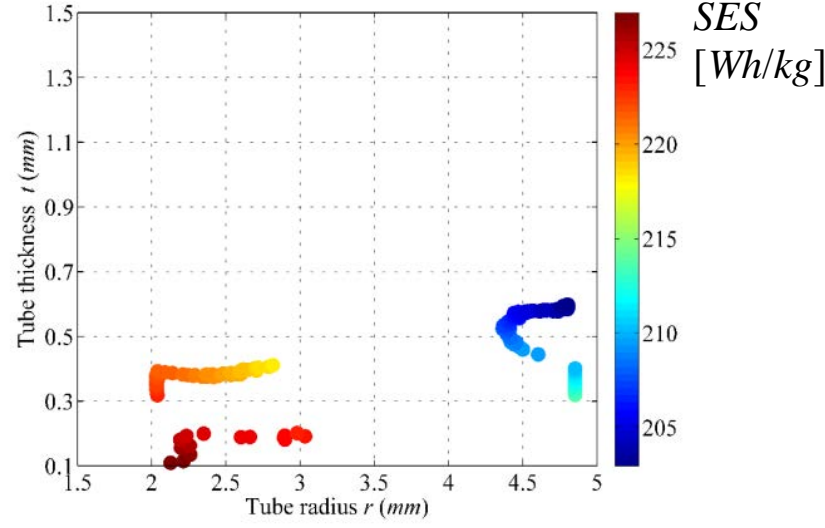

(b)

Figure 16. Representation of optimal solutions in the design variable space. The color-coded information depict the corresponding magnitude of (a) $S E A[\mathrm{~kJ} / \mathrm{kg}]$, and (b) $S E S[\mathrm{Wh} / \mathrm{kg}]$.

Correlations among performance indices, $S E A, S E S, F_{C}$ and $\varepsilon_{\lim }$, are studied to assist the selection of a final configuration. With data from the Pareto solutions a correlation matrix showing correlation coefficients, histograms and anthill plots of performance indices is constructed in Figure 17. The correlation coefficient between SEA and SES is -0.98 which again indicates a strong correlation and competition of the two objectives. Much weaker correlations are found for other pairs of indices making the correlation analysis not very conclusive.

Overall trends show, yet qualitatively, that an increase in $S E A$ corresponds to a gain in $F_{C}$ and $\varepsilon_{\lim }$, whereas an increase in $S E S$ leads to a reduction in $F_{C}$ and $\varepsilon_{\lim }$. For that reason, a designer would not select the maximum SEA as it is associated with poor performance in other aspects. Instead, regarding the safety of batteries and energy absorption performance of CBA, initial collapse load level $F_{C}$ of sacrificing tubes is selected to be the selection criterion in this study. With this consideration, among optimal configurations of similar performance the one with lower value of $F_{C}$, thus providing better protection for batteries, is preferred. Moreover, of the same energy absorption performance, configurations with lower $F_{C}$ have larger deformation. Being a secondary preference, larger tube radius to enhance the cooling performance of CBA is prioritized. Such option allows for more air/coolant fluid flow in the tubes to control temperature of batteries.

The configuration associated with a "knee" point of the Pareto frontier is among potential candidates for the final configuration. When moving from right to left along the Pareto frontier (cf. Figure 15) to approach a "knee-like" point, a slight decrease in SEA leads to a significant increase in $S E S$. Such compromise between SEA and SES makes this "knee-like" point attractive. This point is, however, not considered as a true "knee" point in a strict sense as it does not represent a sharp bend in the Pareto front. To further investigate whether this "knee-like" point is a satisfactory solution, comparison of its initial collapse load level and those of other configurations is made. The correlation of $F_{C}$ and $S E A$ of all optimal solutions is plotted in Figure 18 (a). As stated earlier, configurations with low $F_{C}$ are preferred because they keep batteries safer by facilitating the tube collapse mechanism. The "knee-like" point is, however, associated with a rather high $F_{C}$. Therefore, it should not be chosen as the final configuration.

Using the information of correlations between performance indices in Figure 17, another optimal solution which possesses low $F_{C}$ and moderate $S E A$ is selected, cf. Figure 18 (a). The point 
is graphically determined by using a simple geometry analysis of the $F_{C}-S E A$ correlation. The final configuration corresponds to $r=4.853 \mathrm{~mm}$ and $t=0.3658 \mathrm{~mm}$. With this selection CBA has a relatively high SES $(212 \mathrm{Wh} / \mathrm{kg})$ and moderate $S E A(0.99 \mathrm{~kJ} / \mathrm{kg})$. Moreover, the maximum allowed value of tube radius promotes flow of cooling fluid in tubes to effectively control temperature of batteries. In addition, temperature control of batteries is at its best performance with the maximum tube radius. Finally, the chosen final configuration of CBA can achieve a relatively high deformation level, $\varepsilon_{\lim } \approx 15 \%$, as shown in Figure $18(\mathrm{~b})$.

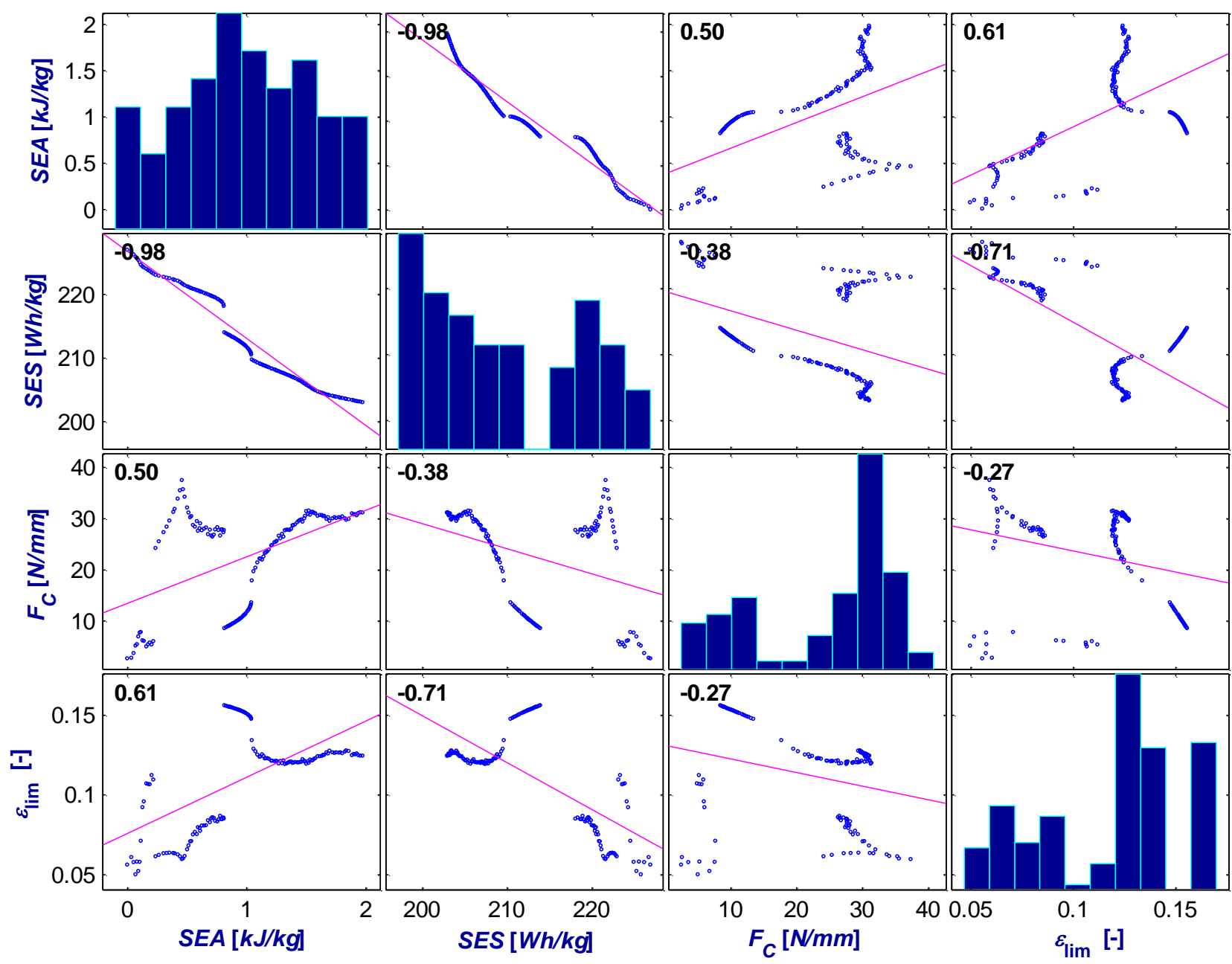

Figure 17. Correlation matrix obtained with data from the Pareto solutions showing correlation coefficients, histograms and anthill plots of performance indices.

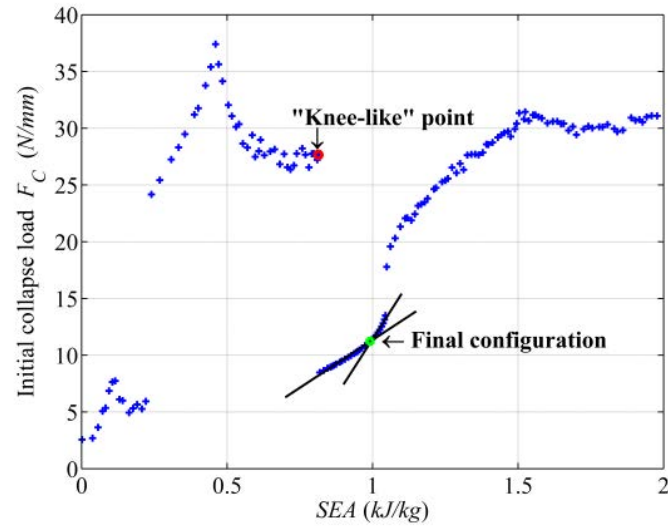

(a)

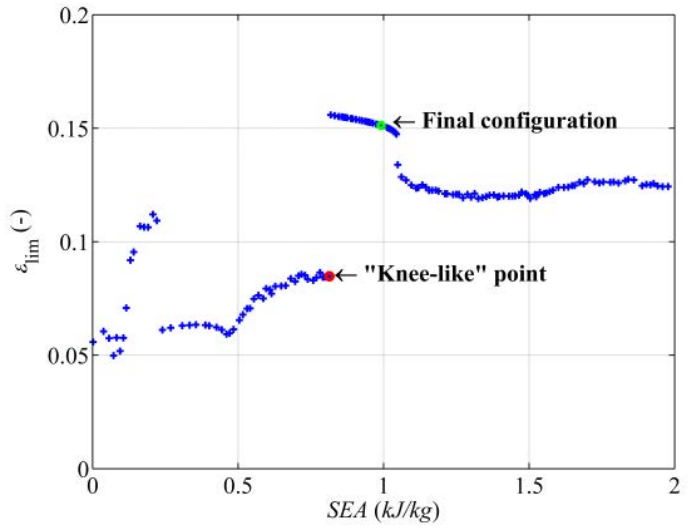

(b) 
Figure 18. Variation of (a) initial collapse load and (b) with respect to $S E A$ of the Pareto optimal solutions.

\section{CONCLUSIONS}

Multi-functionality of a novel architectured battery material system was proposed in this paper. The concept was to integrate the energy storage capability of batteries and energy absorption characteristics of collapsing tubes into a multifunctional Cellular Battery Assembly (CBA). A design procedure including multiple steps to find optimal configurations of CBA was established. While maintaining the battery safety, the optimal design of CBA involves maximizing both energy storage and energy absorption capacities. The trade-off between energy storage and energy absorption of CBA was assessed. Application of an evolutionary approach and metamodeling technique was strategized to successfully find Pareto optimal solutions of the bi-objective optimization problem. A final and most satisfactory configuration with balanced energy storage and energy absorption characteristics was identified when using the initial collapse load as a selection criterion. The final configuration can achieve high energy storage, moderate energy absorption, low collapse load, and high deformation level while maintaining the safety of batteries. With all these features CBA is envisioned to find applications in electrical vehicles. CBA is expected to require less protection from supporting structures and parasitic components compared to that of conventional battery, thereby leading to an overall vehicle weight savings. Also, the energy absorption capability enables CBA to be installed in crumple zones and thus enhance the energy storage capacity of electric vehicles.

\section{ACKNOWLEDGMENT}

This research was supported by the U.S. Department of Energy, Advanced Research Projects Agency-Energy, under contract No. DE-AR0000378. T. Siegmund also acknowledges support from the National Science Foundation (N.S.F.) while working at N.S.F. T. Nguyen acknowledges collaborations with Dr. Nirupam Chakraborti (IIT Khraragpur) through which a customized PPGA tool was made available.

\section{APPENDICES}

\section{A1. Unit cell model}

FE models of the RVE are built in the commercial software ABAQUS [44] as shown in Figure A.1. The RVE is simplified in FE simulations thanks to the symmetry conditions, only a quarter of the RVE is modeled. Analytical rigid surfaces are used to represent the batteries. This conservative condition implies that batteries are not allowed to deform. The continuum plane strain 4-node element with reduced integration option, CPE4R, is used to model the deformable tubes.

A prescribed displacement in vertical and downward direction $\left(U_{Y}\right)$ is applied to the upper battery while the lower one is fixed. Constraint in lateral motion of the batteries can be achieved by adhesively bonding batteries. In addition, electrical bus bars provide further mechanical constraint. The $X$-symmetry boundary conditions are applied to the left and right sides of the model. In addition, contact interactions between the sides and tubes' surfaces are assigned to fulfill the periodicity condition of CBA. Frictionless contact conditions are assigned to model battery-to-tube, tube-to-tube interactions, and self-contact of tubes. All contacts are defined using the available general contact capability in ABAQUS. The dynamic explicit solver ABAQUS/Explicit solver is used to simulate the problem. Reaction force of the fixed battery and displacement of the moving battery are recorded in history outputs.

\section{A2. Construction of a metamodel to approximate $S E A$}

An initial set of 120 sampling points are generated using the LHS method. FE simulations of the RVE model for the whole set are conducted. The load-displacement relation is obtained for each sampling point. The $S E A$ is then computed from the area under the load-displacement curve up to the failure load and the mass of the corresponding configuration. The calculated SEA results at the sampling points are used for the construction of a metamodel using the EvoNN approach. GA parameters applied in the PPGA tool are provided in Table A3. After being constructed, the metamodel is used to approximate the $S A E$ outputs for any set of input. Figure A.2 provides a 
visualization of the computed SEA values and their approximation using the metamodel. The tested and validated metamodel captures pretty well the variation of $S E A$ in the design space.

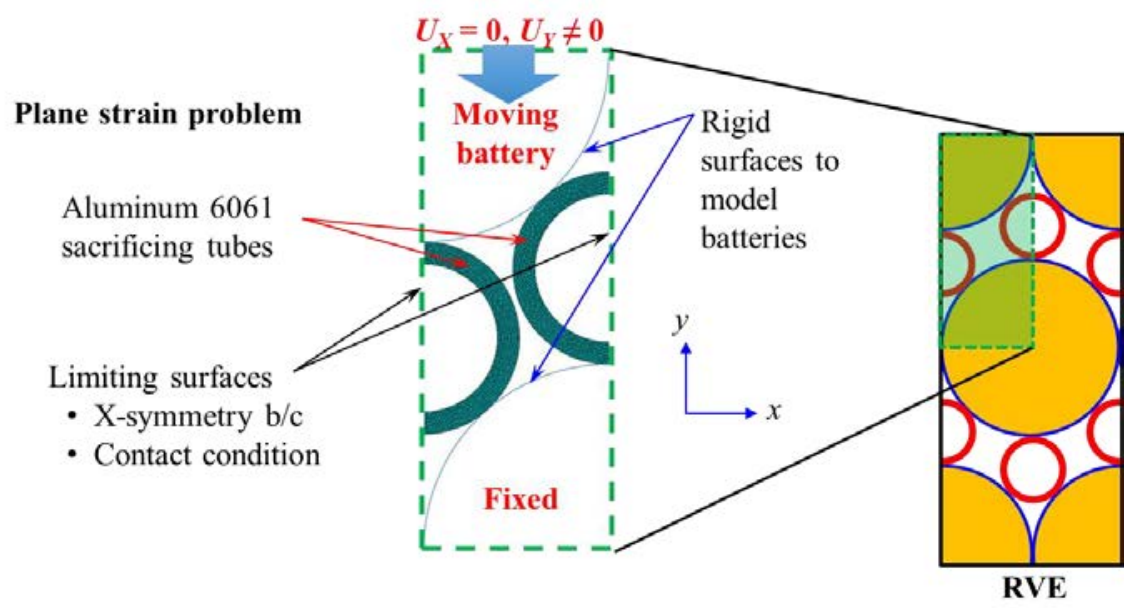

Figure A.1. FE model of the RVE with applied boundary conditions and loading.

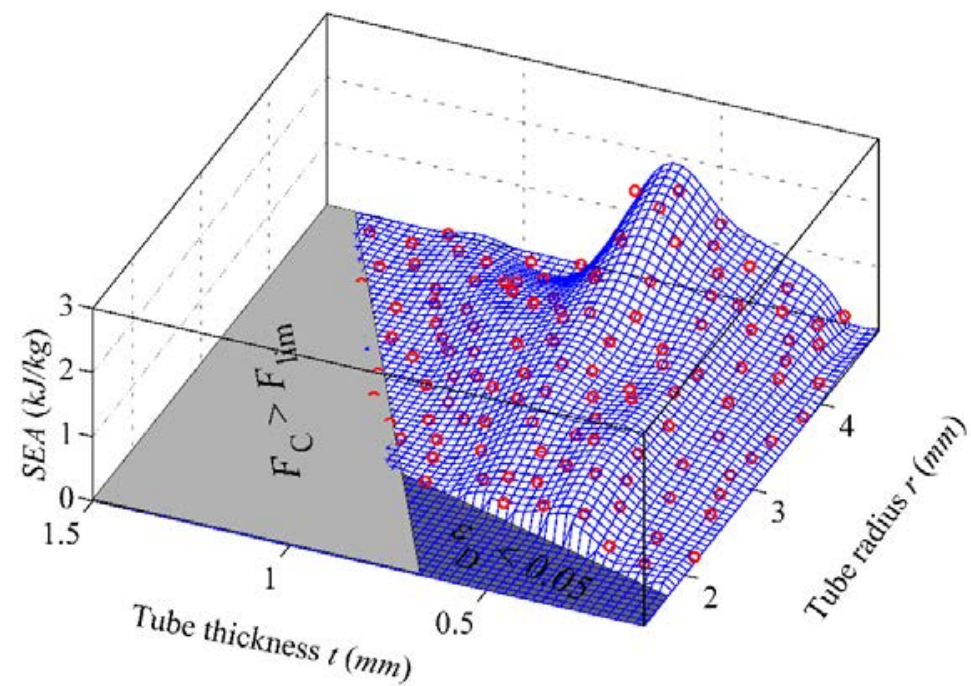

Figure A.2. Variation of SEA in the design parameter space using the constructed metamodel. Symbols represent results of FE simulations at sampling points. Filled areas on the plane $z=0$ represent the constraints in (14) and (15).

Table A4: GA parameters applied in the PPGA code.

\begin{tabular}{|l|c|}
\hline \multicolumn{1}{|c|}{ Parameter } & Value \\
\hline Lattice size & $50 \times 50$ \\
\hline No. of predators & 50 \\
\hline No. of prey (target) & 2000 \\
\hline Probability of crossover & 0.8 \\
\hline Probability of mutation & 0.3 \\
\hline Mutation constant & 0.7 \\
\hline Probability of prey movement & 0.3 \\
\hline Maximum steps for prey movement & 10 \\
\hline No. of hidden layers & 1 \\
\hline Maximum number of nodes in the hidden layer & 50 \\
\hline Probability of omitting a node in the initial population & $0.3-0.99$ \\
\hline
\end{tabular}




\begin{tabular}{|l|c|}
\hline \multicolumn{1}{|c|}{ Parameter } & Value \\
\hline Upper bound for randomly generated weights & 5 \\
\hline Lower bound for randomly generated weights & -5 \\
\hline
\end{tabular}

\section{REFERENCE}

[1] Global EV Outlook: Understanding the electric vehicle landscape to 2020. International Energy Agency: International Energy Agency; 2013.

[2] Delucchi MA, Yang C, Burke AF, Ogden JM, Kurani K, Kessler J, et al. An assessment of electric vehicles: technology, infrastructure requirements, greenhouse-gas emissions, petroleum use, material use, lifetime cost, consumer acceptance and policy initiatives. Philosophical Transactions of the Royal Society of London A: Mathematical, Physical and Engineering Sciences. 2014;372.

[3] Christodoulou L, Venables JD. Multifunctional material systems: the first generation. JOM. 2003;55:39-45.

[4] Snyder J, Gienger E, Wetzel E. Performance metrics for structural composites with electrochemical multifunctionality. Journal of Composite Materials. 2015;49:1835-48.

[5] Wang X, Fan F, Wang J, Wang H, Tao S, Yang A, et al. High damage tolerance of electrochemically lithiated silicon. Nature Commununication. 2015;6.

[6] Tsutsui W, Nguyen T, Liao H, Parab N, Kukreja J, Siegmund T, et al. Mechanical energy dissipation in a multifunctional battery system (in print). MRS Advances. 2016;10.1557/adv.2016.39.

[7] Singh AK, Cao L, Ma J, Seo J, Bakis CE, Zhang Y, et al. Design, manufacture and test of a novel structural battery based on sandwich construction. Journal of Sandwich Structures and Materials. 2015;17:666-90.

[8] Thomas JP, Qidwai M. The design and application of multifunctional structure-battery materials systems. JOM. 2005;57:18-24.

[9] Lyman PC, Feaver TL. PowerCore combining structure and batteries for increased energy to weight ratio. Aerospace and Electronic Systems Magazine, IEEE. 1998;13:39-42.

[10] STORAGE. Car body parts that store electricity, http://ec.europa.eu/research/infocentre/article_en.cfm?id=/research/star/index_en.cfm?p=sS-

storage\&item $=$ Infocentre\&artid $=29520$ (retreived on 03/11/2015).

[11] Roberts SC, Aglietti GS. Structural performance of a multifunctional spacecraft structure based on plastic lithium-ion batteries. Acta Astronautica. 2010;67:424-39.

[12] Schwingshackl CW, Aglietti GS, Cunningham PR. Parameter optimization of the dynamic behavior of inhomogeneous multifunctional power structures. AIAA Journal. 2006;44:2286-94.

[13] Wargnier H, Kromm FX, Danis M, Brechet Y. Proposal for a multi-material design procedure. Materials \& Design. 2014;56:44-9.

[14] Gibson LJ, Ashby MF. Cellular solids: structure and properties. 2nd ed. Cambridge, U.K.: Cambridge University Press; 1999.

[15] Chakraborti N. Promise of multiobjective genetic algorithms in coating performance formulation. Surface Engineering 2014;30:79-82.

[16] Miettinen K. Some methods for nonlinear multi-objective optimization. In: Zitzler E, Thiele L, Deb K, Coello Coello C, Corne D, editors. Evolutionary multi-criterion optimization: Springer Berlin Heidelberg; 2001. p. 1-20.

[17] Coello Coello C, Lamont GB, Veldhuizen DA. Evolutionary algorithms for solving multiobjective problems. 2 ed. New York: Springer US; 2007.

[18] Li X. A real-coded predator-prey genetic algorithm for multiobjective optimization. In: Fonseca CM, Fleming PJ, Zitzler E, Thiele L, Deb K, editors. Evolutionary Multi-Criterion Optimization: Springer Berlin Heidelberg; 2003. p. 207-21.

[19] Giri BK, Hakanen J, Miettinen K, Chakraborti N. Genetic programming through bi-objective genetic algorithms with a study of a simulated moving bed process involving multiple objectives. Applied Soft Computing. 2013;13:2613-23. 
[20] Ashby MF. Multi-objective optimization in material design and selection. Acta Materialia. 2000;48:359-69.

[21] Brechet Y, Bassetti D, Landru D, Salvo L. Challenges in materials and process selection. Progress in Materials Science. 2001;46:407-28.

[22] Ashby M. Designing architectured materials. Scripta Materialia. 2013;68:4-7.

[23] Pettersson F, Chakraborti N, Saxén H. A genetic algorithms based multi-objective neural net applied to noisy blast furnace data. Applied Soft Computing. 2007;7:387-97.

[24] Fonseca CM. Multiobjective genetic algorithms with applications to control engineering problems. Sheffield, UK: Ph.D. Thesis, University of Sheffield; 1995.

[25] Chakraborti N. Chapter 5 - Evolutionary data-driven modeling. Informatics for Materials Science and Engineering. Oxford: Butterworth-Heinemann; 2013. p. 71-95.

[26] Yang RJ, Wang N, Tho CH, Bobineau JP, Wang BP. Metamodeling development for vehicle frontal impact simulation. Journal of Mechanical Design. 2005;127:1014-20.

[27] Nguyen N-T, Hariharan K, Chakraborti N, Barlat F, Lee M-G. Springback reduction in tailor welded blank with high strength differential by using multi-objective evolutionary and genetic algorithms. Steel Research International. 2015;86:1391-402.

[28] Hariharan K, Nguyen N-T, Chakraborti N, Lee M-G, Barlat F. Multi-objective genetic algorithm to optimize variable drawbead geometry for tailor welded blanks made of dissimilar steels. Steel Research International. 2014;85:1597-607.

[29] Hariharan K, Nguyen N-T, Chakraborti N, Barlat F, Lee M-G. Determination of anisotropic yield coefficients by a data-driven multiobjective evolutionary and genetic algorithm. Materials and Manufacturing Processes. 2014;30:403-13.

[30] Ghiabakloo H, Lee K, Kazeminezhad M, Kang BS. Surrogate-based Pareto optimization of annealing parameters for severely deformed steel. Materials \& Design. 2016;92:1062-9.

[31] Tammareddi S, Sun G, Li Q. Multiobjective robust optimization of coronary stents. Materials \& Design. 2016;90:682-92.

[32] Kleijnen JC. Design and analysis of simulation experiments. New York: Springer US; 2008.

[33] McKay MD, Beckman RJ, Conover WJ. A comparison of three methods for selecting values of input variables in the analysis of output from a computer code. Technometrics. 1979; 21:239-45.

[34] Panasonic. NCR18650A, https://na.industrial.panasonic.com/products/batteries/rechargeablebatteries/lithium-ion/series/cylindrical-series/CS474/model/NCR18650A (retreived on 10/28/2015).

[35] Fotouhi A, Auger DJ, Propp K, Longo S, Wild M. A review on electric vehicle battery modelling: from Lithium-ion toward Lithium-Sulphur. Renewable and Sustainable Energy Reviews. 2016;56:1008-21.

[36] Zhu D, Mobasher B, Rajan S, Peralta P. Characterization of dynamic tensile testing using aluminum alloy 6061-T6 at intermediate strain rates. Journal of Engineering Mechanics. 2011;137:669-79.

[37] Nguyen NT, Kim DY, Kim HY. A continuous damage fracture model to predict formability of sheet metal. Fatigue \& Fracture of Engineering Materials \& Structures. 2013;36:202-16.

[38] Heppes A. Some densest two-size disc packings in the plane. Discrete and Computational Geometry. 2003;30:241-62.

[39] Kennedy T. Compact packings of the plane with two sizes of discs. Discrete and Computational Geometry. 2006;35:255-67.

[40] Ashby MF. Overview No. 80: On the engineering properties of materials. Acta Metallurgica. 1989;37:1273-93.

[41] Salimon A, Bréchet Y, Ashby MF, Greer AL. Selection of applications for a material. Advanced Engineering Materials. 2004;6:249-65.

[42] Reid SR. Laterally compressed metal tubes as impact energy absorbers. In: Jones N, Wierzbicki T, editors. Structural Crashworthiness. London: Butterworths; 1983. p. 1-43.

[43] Evans AG, Hutchinson JW, Ashby MF. Cellular metals. Current Opinion in Solid State and Materials Science. 1998;3:288-303.

[44] Simulia. ABAQUS 6.13 Analysis Manual. 2012. 


\section{Graphical Abstract}

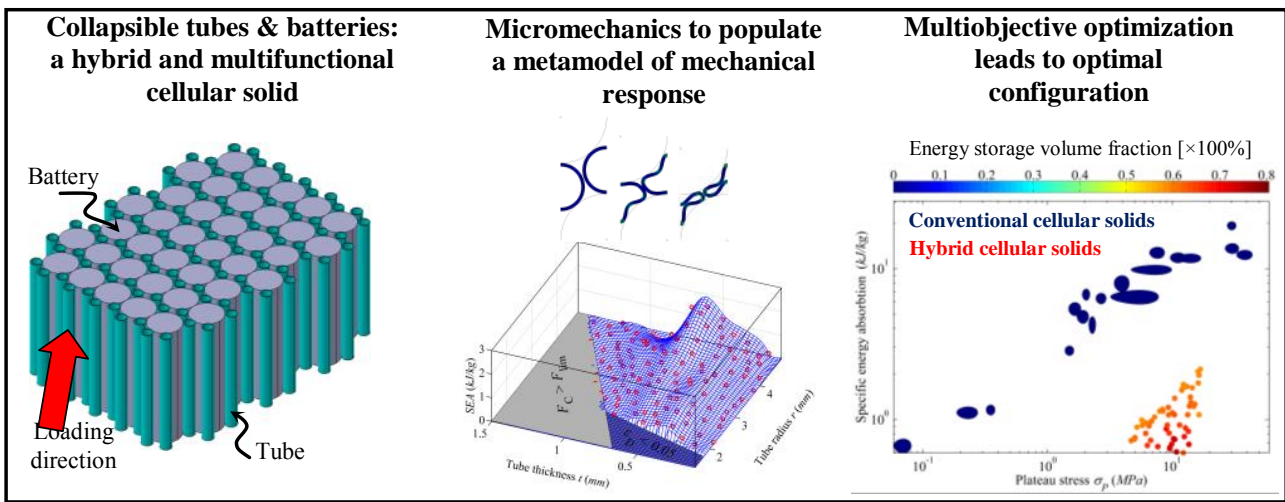

